Article

\title{
Detection and Localization of Interference and Useful Signal Extreme Points in Closely Coupled Multiconductor Transmission Line Networks
}

\author{
Ruslan R. Gazizov ${ }^{1, *} \mathbb{D}$, Timur T. Gazizov ${ }^{2}$ and Talgat R. Gazizov ${ }^{1}$ \\ 1 Television and Control Department, Tomsk State University of Control Systems and Radioelectronics, 634050 \\ Tomsk, Russia; talgat@tu.tusur.ru \\ 2 Information Technologies Department, Tomsk State Pedagogical University, 634061 Tomsk, Russia; \\ timurtsk@gmail.com \\ * Correspondence: ruslangazizow@gmail.com; Tel.: +7-3822-90-01-45
}

Received: 28 August 2019; Accepted: 26 September 2019; Published: 27 September 2019

\begin{abstract}
This study highlights the importance of detecting and localizing useful and interference signal extreme points in multiconductor transmission lines (MCTL) by developing a new approach for detecting and localizing signal extreme points in MCTL networks of arbitrary complexity. A radio-electronic component is presented as a network consisting of a number of connected MCTL sections. Each MCTL section is divided into segments and the number of segments is set by the user. The approach is based on a quasi-static calculation of signal waveforms at any point (segment) along each conductor of an MCTL. The block diagrams of the developed algorithms are presented. Using the approach, a number of investigations have been done which include the following: the signal maximum detection and localization in the meander lines with one and two turns, the influence of ultrashort pulse duration on localization of its extreme points in the printed circuit board (PCB) bus of a spacecraft autonomous navigation system, the influence of ultrashort pulse duration on localization of crosstalk extreme points in the PCB bus, and the simulation of electrostatic discharge effects on the PCB bus. There are also some investigations with optimization methods presented. A genetic algorithm (GA) was used to optimize the influence of ultrashort pulse duration on localization of the pulse and crosstalk extreme points in the PCB bus. Furthermore, the GA was used to optimize the PCB bus loads by criteria of the peak voltage minimization. A similar investigation was carried out with the evolution strategy. The obtained results help us to argue that the signal extreme points can be detected both in structures with different configurations and applying different excitations.
\end{abstract}

Keywords: computer simulation; diagnostics of radio-electronic equipment; electrostatic discharge; evolution strategies; genetic algorithms; interference; optimization; quasi-static analysis; signal extreme points; spacecraft navigation system; ultrashort pulse

\section{Introduction}

The increasing complexity of devices being developed and physical processes occurring in them make a mathematical simulation very important, in particular, because a full-scale simulation is more expensive than a mathematical simulation. Moreover, a lot of conductors create various crosstalk noises and parasitic couplings which complicate the process of diagnosing radio-electronic equipment (REE) because of the necessity to take into account all couplings between conductors [1]. It is important for the diagnostics to be carried out at the designing stage and be aimed at detecting possible vulnerabilities of printed circuit boards (PCB), for example, detecting the places with an excess of voltage or current amplitudes [2,3]. Timely and accurate application of a mathematical simulation would exclude the influence of electromagnetic interference (EMI) on high sensitive equipment during 
its operation [4], as well as decrease the consequences of intentional EMI which make the REE inoperative [5,6]. Ultra-wideband pulses, power ultrashort pulses [7], and electrostatic discharge (ESD) [8] can be referred to as such excitations. Due to this fact, it is necessary to take into account both useful and interference signals during the PCB diagnostics. Moreover, it is particularly important in the field of aerospace because a fault that appears in one of the parts of aircraft equipment can cause the loss of an aircraft $[9,10]$. For example, the new method of hardware-in-the-loop simulation for an aircraft electric power system has been presented in [11] and the use a new fault diagnostic method by multi sensor fusion for testing spacecraft control system sensors has been proposed in [12]. In addition, the noises (crosstalk) generated by the useful signals, the powerful intentional electromagnetic pulses of nanosecond and subnanosecond ranges, are dangerous because they are capable of penetrating into various electronic equipment and disabling them. This is important for supporting the uninterrupted operation of critical systems, for example, to assess the situation of electromagnetic terrorism manifestations that have powerful electromagnetic effects on the electrical network, or to protect special technical buildings from external electromagnetic effects. The propagation of such signals in multiconductor transmission lines has been well studied, however, the features of the ultrashort pulse propagation along the conductors with a high density of PCB interconnects are not clearly understood and can lead to their uncontrolled propagation, whereby it is important to provide the control of signal propagation along the entire length of the conductors.

The way to determine the propagation path of noise using the source modulation technique was reviewed in [2], which considered the key parameters and the main properties of the noise source modulation technique (NSMT) based on the amount and the type of modulation of the noise at the victim location. The experiment was performed under ideal conditions, in which two cables had the same common-mode currents, but only one of them was a noise propagating path for the victim. By comparing the propagation path index with the measured insertion loss between the cables and receiving antennas, the NSMT was proved to determine the noise propagation path. Quantifying EMI was examined in [3], namely a methodology for determining and quantifying radiation for practical design guidelines. Due to tight EMC standards, it becomes increasingly important to diagnose the radiation hot spot in the radiation process before applying attenuation measures. The researchers implemented the analysis of characteristic mode together with the integral equation based numerical method in order to identify the hot spots for EMI radiation. Several numerical examples were applied to benchmark the proposed approach. An analysis in time domain and simulation of crosstalk noise reduction for high-speed digital circuits by a guard trace with an open stub between coupled striplines was carried out in [13]. In order to mitigate the interference of crosstalk noise in PCBs or packages the grounded guard traces were utilized. The study included a discussion on how the guard trace with inserted coupled striplines with an open stub affects the crosstalk noise. A design curve for the geometric parameters of coupled striplines inserted guard trace stub was constructed based on the proposed method. The maximum reduction in crosstalk noise in the presented examples exceeded $50 \%$. A new method for the fast-transient analysis of large linear chains using stable high-order methods was presented in [14]. A new algorithm based on the A-stable and L-stable high-speed integration methods in the time domain for simulating large linear circuits was presented. The proposed method took advantage of the mathematical description of the circuits found in these cases in a special form to significantly reduce computational costs. Several variants of schemes were presented to demonstrate the acceleration achieved by the proposed algorithm.

The optimization by means of different methods based on collective intelligence to different application areas has been widely used. For example, a genetic algorithm (GA) with real number encoding was used for structural damage detection based on vibration data in [15]. The objective function was minimized by directly comparing the changes in the measurements before and after damage. Three different criteria were considered, namely, the frequency changes, the mode shape changes, and their combination. All the damaged elements could be detected accurately by genetic algorithm, as seen from the numerical results, even when the analytical model was not accurate. A 
particle swarm optimization algorithm was used for the quantization thresholds in [16]. The authors considered decentralized detection of an unknown signal corrupted by zero-mean unimodal noise via wireless sensor networks. The asymptotic performance analysis of the multi-bit Rao test was provided and exploited to propose a (signal independent) quantizer design approach by maximizing the noncentrality parameter of the test statistic distribution. Numerical results were provided to show the effectiveness of the Rao test in comparison to generalized likelihood ratio test and the boost in performance obtained by (multiple) threshold optimization. A GA was used for mobile robot localization using ultrasonic sensors in [17]. The researchers investigated an ultrasonic sensor localization system for autonomous mobile robot navigation in an indoor semi-structured environment. They developed a self-localization module which has been integrated successfully in a more complex navigation system. A method for the joint optimization of radio and computing resources for multichannel mobile computing was proposed by [18]. This study, considered a multiple-input multiple-output (MIMO) multicell system where several mobile users ask for computation offloading to an ordinary cloud server. The resulting optimization problem was nonconvex. Nevertheless, in the case of a single user, researchers were able to calculate the main optimal solution in closed form. In the more challenging scenario with an increasing number of users, they suggested an iterative algorithm, which was based on a novel successive convex approximation approach, converging to a local optimal solution of the original nonconvex task. Numerical results showed that the proposed approach excelled the other optimization methods. The projection matrix was optimized for sparse signals in structured interference in [19]. The researchers considered the problem of estimating a signal which has been corrupted with structured noise and proposed an approach for the design optimization of the projection matrix, in which the objective was not only to decrease the amount of data to be processed but also to eliminate the undesired signal components. As a result, they reduced the computation time and the error on the estimation of the unknown parameters of the sparse model, with respect to the uncompressed data. The joint optimization of data and energy transfer in multiuser MIMO systems was performed in [20]. The researchers presented a method to solve the nonconvex optimization task that appeared when constructing the transmit covariance matrices in multiuser MIMO broadcast networks with implementation of simultaneous wireless information and power transfer. Two different approaches were applied to reformulate the (nonconvex) multiobjective problem. In order to solve the resulting formulations, the researchers proposed using the majorization-minimization approach. A method for optimizing the received signal strength (RSS) using the Gauss method was presented in [21]. The optimal RSS threshold was found through minimizing the best achievable localization root mean square error formulated with the aid of fundamental lower bound analysis. The resulting optimal RSS threshold enabled enhanced performance of new fashioned low-cost and low-complex proximity report-based positioning algorithms. A synthesis of a control system based on multicriteria optimization using a GA was presented in [22] in which an approach for the parametric synthesis of the automatic control system (ACS) was suggested. The approach used the genetic algorithm that carries out an approximation of Pareto-optimal solution sets and it was shown that their approach obtained a reasonable solution in a shorter time and with less computational costs than other methods. In [23] optimization was considered using a GA, optimization by the particle swarm method and optimization by the firefly algorithm when designing antennas, and demonstrated the application of the evolutionary optimization methods with multiple objectives to the microstrip antennas constructing. Impedance matching and gain improvement were optimized over a predefined frequency range, resulting in a very small and compact $12 \mathrm{~mm} \times 21 \mathrm{~mm}$ ultra-wideband antenna which was fabricated and measured. For radio-electronic equipment optimization the most popular are GAs and evolution strategies (ES) [24]. For example, a new algorithm for optimizing a direct eye diagram for an arbitrary transmission line using a finite impulse response (FIR) filter was proposed in [25] in which a new design algorithm, which directly optimizes the eye diagram using the FIR filter as transmitter pre-emphasis, was proposed to counteract the intersymbol interference in the high-speed data transmission. In order to minimize the resistance of electrical contact, a method for controlling the location of microcontacts based on a 
GA was proposed in [26]. In this study, the positioning and sizing optimization of microcontact spots were investigated in relation to minimizing the ECR. The optimal solutions were obtained by a novel method of a real-coded genetic-algorithm implemented with a subpopulation-based selection method and a normal distribution probability-based crossover. A method for analyzing and optimizing signal integrity for complex circuits with multiple inputs and outputs based on the analysis of S-parameter data was proposed in [27]. The proposed data mining approach considered both the interconnect structure and the stimulated pattern of a MIMO system, which can carry out thoughtful analysis and optimization with high efficiency. The decentralized dynamic optimization of voltage management in the power supply network was investigated in [28]. By simulating the network dynamics using an autoregressive process and considering time-varying resource constraints, the researchers provided an error bound in tracking the instantaneous optimal solution to the quadratic error objective. The design of the circuit of the delay lines using a GA to minimize crosstalk noise was investigated in [29]. The paper highlighted a development of a design methodology of delay line layout for minimizing crosstalk with the use of a GA for optimization. The GA required a large number of function evaluations, and efficient calculation of crosstalk was proposed together with a new approach of generating random line, making offsprings, and mutation. Different optimum results have been obtained for different objectives and compared.

The quasi-static approach is widely used for analyzing PCB interconnects, because the accuracy of the circuit analysis is often unacceptable, while the electromagnetic analysis often involves large computational costs. The investigations of techniques for detecting and localizing signal extreme points have been performed for single and coupled $[30,31]$ transmission lines, and for a PCB bus of a spacecraft autonomous navigation system (ANS) [32,33], also with the use of a GA $[34,35]$. The voltage maximum exceeding the signal amplitude at the input by 1.14 times is detected in the meander line with two turns. The voltage maximum, twice as big as the signal amplitude at the input, is detected in the microstrip cross section. The voltage maximum exceeding the steady state level by $18 \%$ is detected in the PCB bus of a spacecraft autonomous navigation system. The voltage maximums exceeding the steady state level by $36 \%$ and $38 \%$ are revealed in the PCB bus by using the GA for optimization. The voltage maximum exceeding the steady state level by $20 \%$ is detected in the PCB bus by using the ES optimization.

However, an approach combining the opportunity to perform the diagnostics for detecting and localizing signal extreme points and the optimization of obtained results has not been developed. Therefore, the purpose of this paper is to develop the approach and to demonstrate some recently obtained and new results of applying this approach in detecting and localizing extreme points of useful and interference signals which are necessary for REE diagnostics.

The paper is organized as following. In Section 2, a brief theory is presented. In Section 3, a description of the proposed approach for detecting and localizing signal extreme points is presented. In Section 4, block diagrams of the developed algorithms for the approach are shown. The results of the investigation of coupled transmission lines are presented in Section 5. In Section 6, the results of the investigation of the spacecraft ANS bus are considered. In Section 7, the results of the GA for optimization are shown. In Section 8, the results of the GA for optimization are shown. Section 9 is the conclusions.

\section{Theory}

The theoretical bases and algorithms for calculating quasi-static responses along each conductor of each multiconductor transmission line (MCTL) section connected to the multiconductor network were developed earlier and presented in [30]. But it seems to be important to show them again for a full understanding of the approach.

Frequency domain equations are used for calculating voltage and current responses in an MCTL section:

$$
\mathbf{V}(x)=\mathbf{S}_{V}(\mathbf{E} 0 \cdot \mathbf{C} 1+\mathbf{E D} \cdot \mathbf{C} 2)
$$




$$
\mathbf{I}(x)=\mathbf{S}_{I}(\mathbf{E} 0 \cdot \mathbf{C} 1-\mathbf{E D} \cdot \mathbf{C} 2)
$$

where $S_{V}$ and $S_{I}$ are the matrices of modal voltages and currents; E0 and ED are the diagonal matrices $\mathrm{E} 0=\operatorname{diag}\left(\exp \left(-\gamma_{1} x\right), \ldots, \exp \left(-\gamma_{N_{k}} x\right)\right)$ and $\mathrm{ED}=\operatorname{diag}\left(\exp \left(-\gamma_{1} \cdot(l-x)\right), \ldots, \exp \left(-\gamma_{N_{k}}(l-x)\right)\right) ; \gamma_{N_{k}}$ is the propagation constant for the $k$-th MCTL section; $N_{k}$ is a number of conductors in the $k$-th MCTL section; $l$ is the length of MCTL section; and $x$ is the coordinate along the MCTL section (the number of $x$ is defined by $n_{\text {TLS }}$ parameter). The calculation of $\mathrm{S}_{V}, \mathrm{~S}_{I}$, and $\mathrm{E}(x)$ is described in [36]. C1 and C2 are constant vectors calculated as:

$$
\left[\begin{array}{l}
\mathbf{C}_{1} \\
\mathbf{C}_{2}
\end{array}\right]=\left[\begin{array}{cc}
\mathbf{S}_{V} & \mathbf{S}_{V} \mathbf{E}(l) \\
\mathbf{S}_{V} \mathbf{E}(l) & \mathbf{S}_{V}
\end{array}\right]^{-1}\left[\begin{array}{c}
\mathbf{V}(0) \\
\mathbf{V}(l)
\end{array}\right]
$$

where $\mathrm{E}(l)=\operatorname{diag}\left(\exp \left(-\gamma_{1} l\right), \exp \left(-\gamma_{2} l\right), \ldots \exp \left(-\gamma_{N_{k}} l\right)\right) ; \mathrm{V}(0)$ and $\mathrm{V}(l)$ are vectors describing the voltage at the ends of the MCTL section, determined after solving the equation for the circuit with $n$ MCTL sections with lumped elements at the ends:

$$
\mathbf{V}=\left(s \mathbf{W}+\mathbf{H}+\sum_{k=1}^{n} \mathbf{D}_{k} \mathbf{Y}_{k} \mathbf{D}_{k}^{t}\right)^{-1} \mathbf{E}
$$

where $s=j \omega$, where $\omega$ is angular frequency; $\mathrm{W}$ and $\mathrm{H}$ are matrices of order $A \times A$ describing the lumped memory and memoryless elements of the network, respectively ( $A$ is the number of parameters, which are calculated by the modified node potential method); $\mathbf{D}_{k}=[\mathrm{i}, \mathrm{j}]$ with entries $l_{i, j} \in\{0,1\}$, where $i \in\left\{1, \ldots, N_{k}\right\}, j \in\left\{1, \ldots, 2 N_{k}\right\}$ with one nonzero value in each column, is the selector matrix that maps the terminal currents of the $k$-th MCTL section; $\mathbf{Y}_{k}$ is the conductance matrix of the $k$-th MCTL section; $\mathrm{V}$ is the vector of the node voltage waveforms; and $\mathrm{E}$ is a constant vector with the entries determined by the independent voltage and current sources.

The description of the response calculation algorithm is presented in [36]. By applying the forward fast Fourier transformation (FFT), the original excitation in the time domain is transformed into the frequency domain. Then, calculations of Equations (1) to (4) are carried out. Then, by applying the inverse FFT, the obtained result is transformed into the time domain.

\section{Proposed Approach for Signal Extreme Points Detection and Localization}

On the basis of the theory presented in Equations (1) to (4), an approach for signal extreme points detection and localization was developed. The description of the approach is presented further. First, an REE developer should make a correct circuit diagram of a structure under development. Then, he should choose a fragment in one of the conductors of the structure to be diagnosed. The diagnostics is aimed at checking whether the amplitudes of a propagating signal exceed the maximum agreed value or how the signal waveform is transformed during the propagation. The chosen fragment can consist of several segments of transmission lines or can be chosen over the whole conductor. In addition, the fragment can be located at any conductor of the structure (the developer can perform the diagnostics of the active or passive conductors to analyze the signal or crosstalk waveforms propagation respectively).

Thus, the proposed approach includes the following stages:

Preparation stage Formulating a problem to be solved, the aim, and expected results. Designing cross sections and a circuit diagram of REE (taking into account all galvanic connections between circuit diagram components), adding all parameters of active and reactive components (for a transmission line section, the per unit length coefficient matrices of electromagnetic $(L)$ and electrostatic $(C)$ induction, conductivities (G), and resistances (R); for the excitation signal source, the type of a signal and its parameters).

Calculation stage Setting up the start and end points of the pulse propagation, the number of a segment along each MCTL section, and the speed of displaying. Calculating signal waveforms. Detecting and localizing signal extreme points. Performing optimization to obtain an optimal solution. 
Final stage Analyzing the obtained results, i.e., signal waveforms, whose propagation along each conductor of each MCTL is displayed in dynamics; detected signal extreme points; localization of these points in the circuit diagram.

Let us consider the calculation and final stages in detail. In the calculation stage, to choose the fragment of the conductor (or the whole conductor) that consists of several MCTL sections, the developer should set the start and end points in the circuit diagram. The diagnostics and visualization of the signal propagation will be done along this fragment. Each MCTL section is divided into a number of segments which are set by the developer. The signal waveform is calculated in each segment of each MCTL section. The response (signal waveforms) calculation algorithm differs from the algorithm described in [36] primarily in the fact that it uses segment coordinates instead of a node number. As a result, the system stores voltage or current values calculated in each segment of each conductor section. Then, the signal propagation way along the chosen conductor is defined taking into account all galvanic connections between MCTL sections (in other words a successive display of the signal waveforms calculated in each MCTL segment). The speed of the display is also defined by the developer. Then, the search for signal extreme points is carried out in each segment. It should be mentioned that we need to find not all extreme points but the global (relative to all segments) maximum and minimum only. The numbers of the segment and the MCTL section are assigned to the global maximum or minimum. These numbers are then used for localizing the extreme points. Moreover, there is an opportunity to apply the GA for optimization in this stage, for example, if a user needs to define such excitation pulse duration at which the maximum value of the signal amplitude along the conductor will be the highest.

In the final stage, dynamic visualization of the signal propagation along the conductor is performed. The developer can control the visualization as follows: stop the animation, display the signal waveform in the previous and next segment, and continue the animation. The developer can also push a button with an extreme point to display the signal waveforms with the global extreme point (maximum or minimum). These results are displayed in another window of the program. At the same time, the segments with the calculated signal waveforms are displayed as points in the circuit diagram (according to the signal waveform animation). The animation in the circuit diagram stops when the signal extreme point is displayed, and therefore one can see the place where the extreme point is located. Thus, the developer can perform the diagnostics aimed at checking what global extreme voltages or currents have been detected, where they are localized, and how the signal waveforms have been transformed during its propagation along the conductor. A block diagram of the proposed approach is presented in Figure 1.

\section{PREPARATION STAGE}

- Defining the problem.

- Designing cross-sections and a circuit diagram.

CALCULATION STAGE

- Setting up the start and end points for visualizing signal propagation.

- Setting up the number of segments.

- Setting up a display speed value.

- Searching for signal extreme points.

- Localizing signal extreme points.

- Performing an optimization (if needed).

\section{FINAL STAGE}

- Displaying signal propagation in dynamics.

- Displaying localized extreme points.

- Analyzing results (signal waveforms, signal extreme point values and locations, optimization results).

Figure 1. Approach for signal extreme points detection and localization. 


\section{Developed Algorithms used in the Approach}

There are three algorithms which were devised while developing the approach. They are the visualization algorithm of signal propagation along the circuit, the algorithm for defining the allowed ways of signal propagation along the circuit, and the algorithm for detecting and localizing signal extreme points.

\subsection{Visualization Algorithm of Signal Propagation along the Circuit}

A block diagram of this algorithm is presented in Figure 2. Let us consider the essence of the algorithm. At first, step 1, you should choose the start node $A$ and the end node $B$ between which the visualization of a signal propagation will be shown. Then, step 2 (considered as the algorithm in detail in Section V) defines the propagation way based on these nodes, a number and also an order of MCTL sections and also an order of its segments. The segments' order will be used in the visualization of signal propagation. As a result of this algorithm, the system generates some commands and performs dynamic visualization.

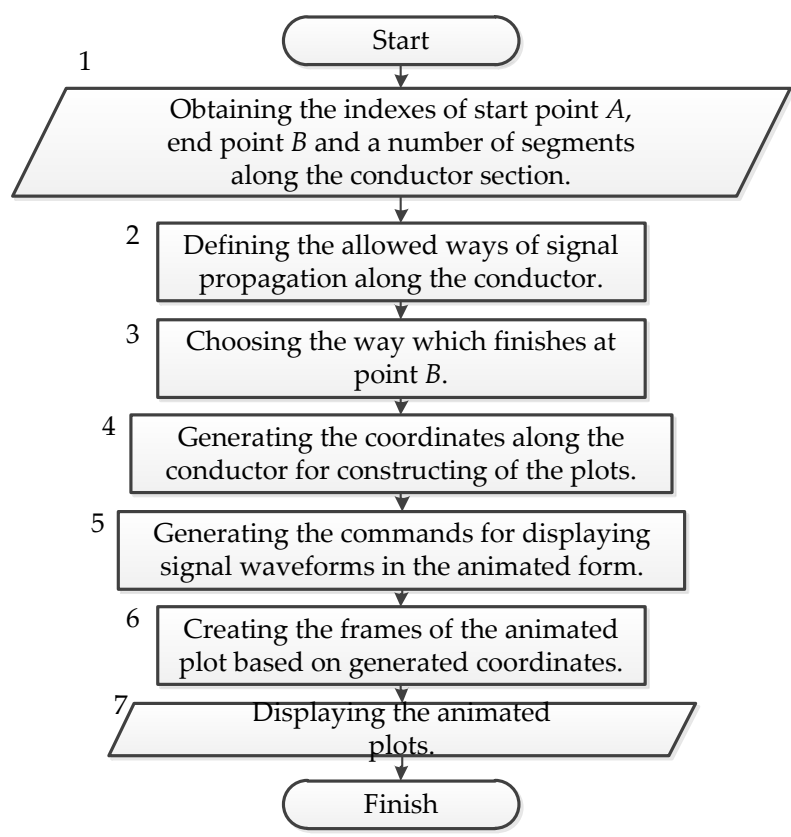

Figure 2. Block diagram of the visualization algorithm of signal propagation along the circuit.

\subsection{Algorithm for Defining the Allowed Ways of Signal Propagation along the Circuit}

A block diagram of this algorithm is shown in Figure 3.

Point $A$ can be chosen at any node of the circuit diagram, but the allowed locations of node $B$ are defined by the algorithm for defining the allowed ways of signal propagation along the circuit. The aim of the algorithm is to provide a condition for maintaining galvanic connections between the MCTL sections on the way of signal propagation. A test circuit diagram is shown in Figure 4. All the parameters of the circuit components are not shown in Figures 4-7 because they are not important in this part. MCTL sections in these Figures are called "trl". The circuit diagram configuration has the primary meaning. Each step of the algorithm before the complete processing of the first circuit component is shown in Figure 5. 


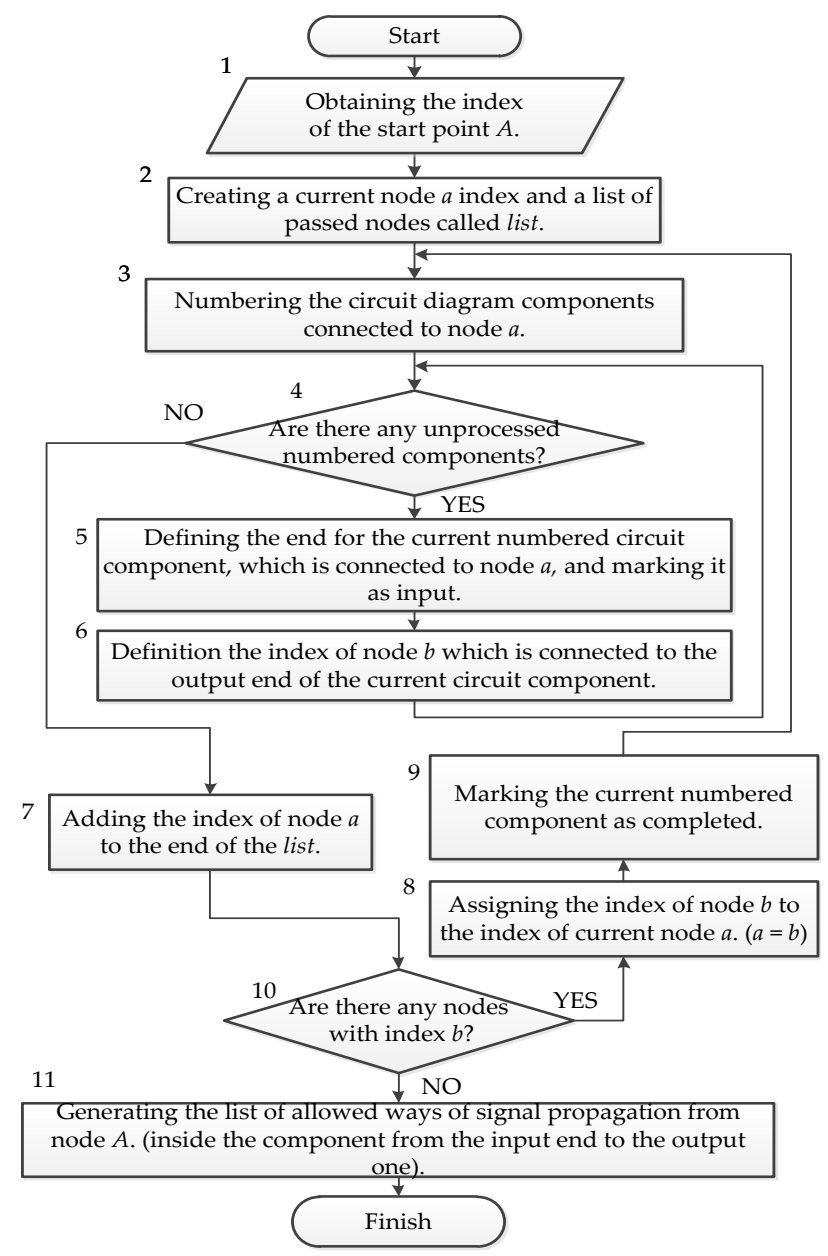

Figure 3. Block diagram of the algorithm for defining the allowed ways of signal propagation along the circuit.

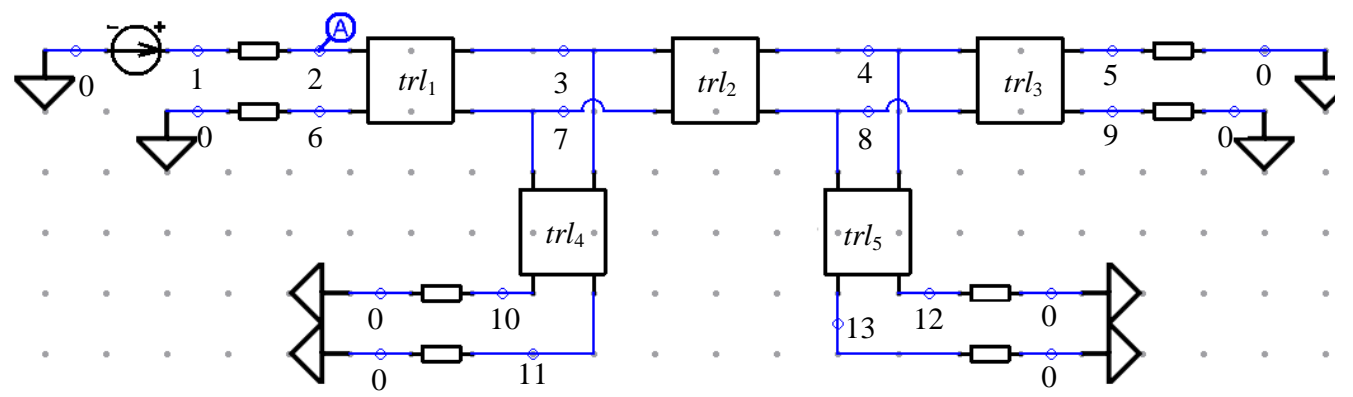

Figure 4. Test circuit diagram. 

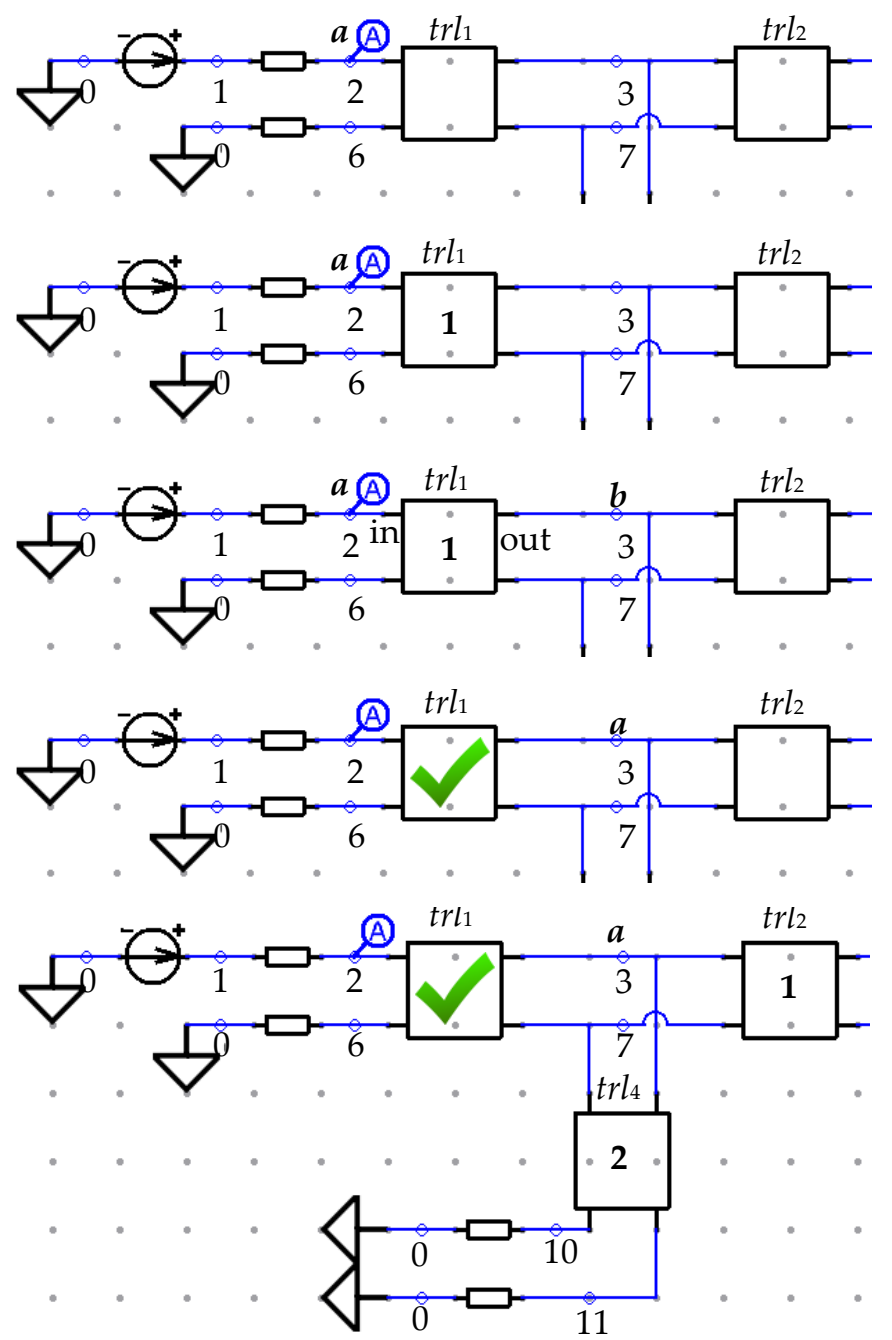

Figure 5. Display of each step of the algorithm before the complete processing of the first circuit component.

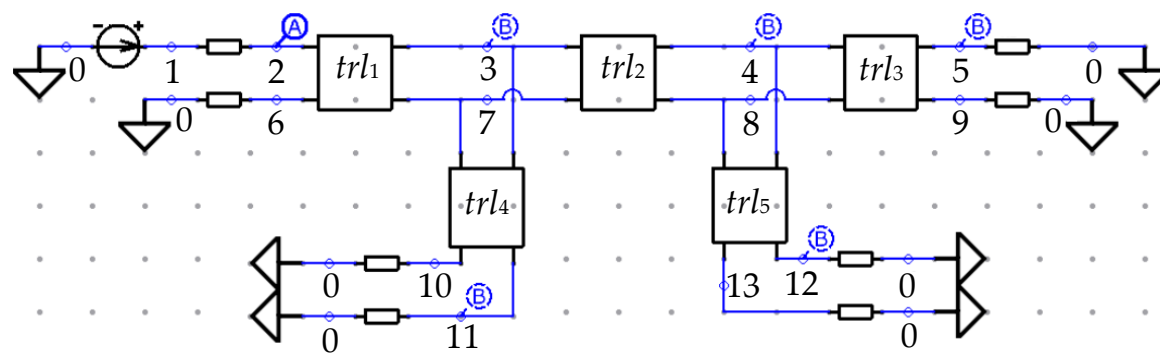

Figure 6. All allowed end nodes $B$ for the test circuit diagram.

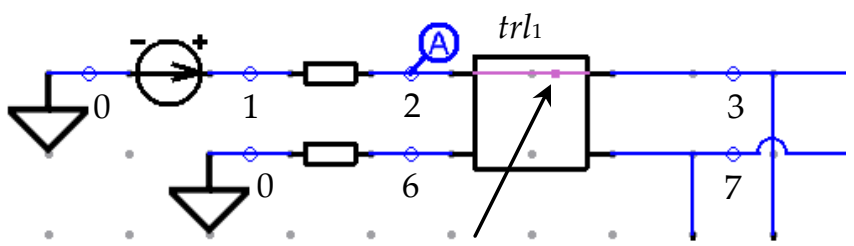

Figure 7. Signal localization example.

Let us consider each step of the algorithm according to the test circuit diagram as follows:

(1) The index of node $A$ appears in the system (when a user chooses it). $A=2$. 
(2) The variable of the list type called "list" is created (empty at first). The start node index is assigned to a current node index $a(a=A=2)$.

(3) The circuit components connected to node $a$ are numbered.

(4) The check of other unprocessed components is performed. If there is an unprocessed component, then go to step 5, otherwise, go to step 7. (Numbers of the steps are shown in Figure 3.)

(5) The processing of each numbered component is performed in succession. The component end connected to the node $a$ is defined as input and the opposite one is defined as output.

(6) The index of the node connected to the output end is assigned to node $b(b=3)$.

(7) The node $a$ index is added to the end of the "list" (list contains 2).

(8) The check of another node with $b$ index is performed. If there is a node with $b$ index, then go to step 9, otherwise, go to step 11 .

(9) There is a node with $b$ index. The index of node $b$ is translated to the node $a$ index $(a=3)$.

(10) The current numbered component $\left(t r l_{1}\right)$ becomes completed (shown by green ticks in Figure 5 ). Other elements connected to node $a$ are numbered again. Therefore, we go to step 3 of the algorithm.

(11) The algorithm will work until nodes $b$ will run out.

At the end of the algorithm run (shown in Figure 3), the designer can choose the end node $B$ by moving the arrow of the mouse (all allowed end nodes $B$ for the test circuit diagram are shown in Figure 6).

MCTL sections are schematically shown as squares in the figures. The list of nodes and MCTL segments (where the signal waveforms should be generated) is created in the system after setting up points A and B. The list is sent to the display module which creates the frames of dynamic visualization of signal propagation along the conductor. A place where the signal waveform is calculated is displayed in the circuit diagram simultaneously with the dynamic visualization. The whole section of the MCTL conductor is highlighted and a colored point shows a certain place along this conductor. An example of signal localization by a colored point in the test circuit diagram is shown in Figure 7.

\subsection{Algorithm for Detecting and Localizing Signal Extreme Points}

The block diagram of the algorithm for detecting and localizing signal extreme points is shown in Figure 8.

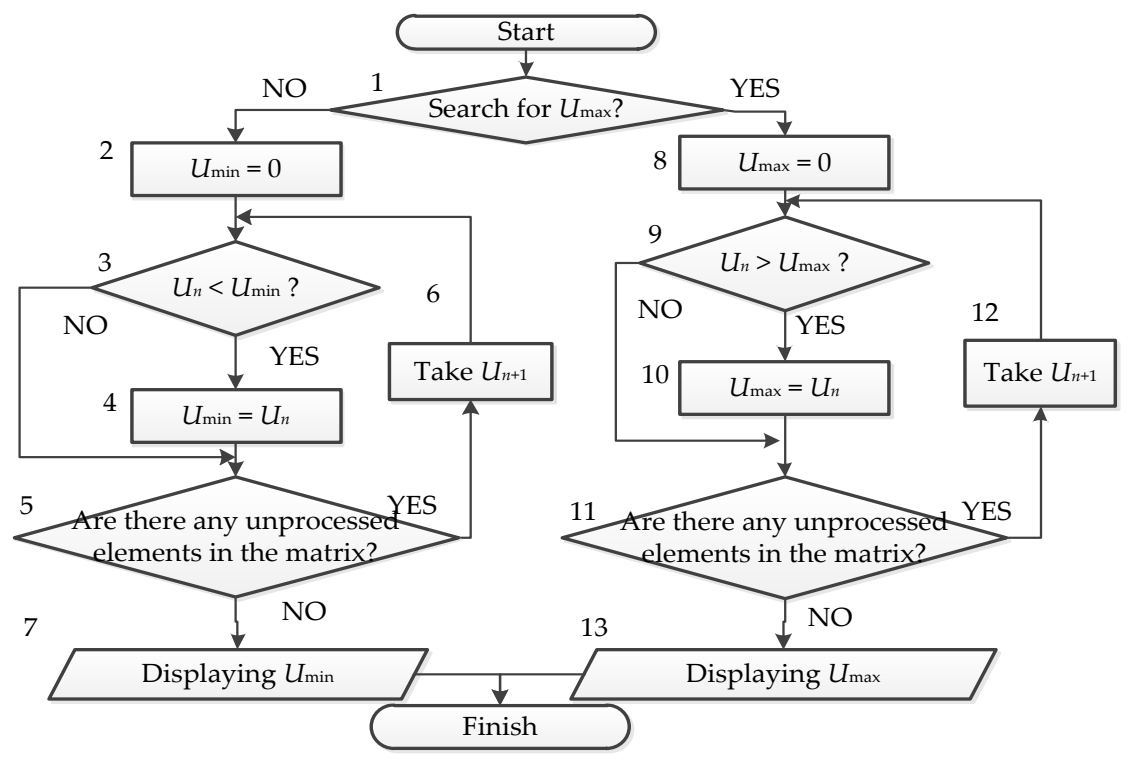

Figure 8. Block diagram of the algorithm for detecting and localizing signal extreme points. 
Let us consider this algorithm in detail. The results of calculating the response in one segment are kept in a matrix consisting of one column. The essence of the algorithm is in the blind search for the maximum or the minimum value in the matrix. All symbolic notations in Figure 8 are for voltages, where $U_{\max }$ is the maximum voltage value, $U_{\min }$ is the minimum voltage value, $U_{n}$ is the current matrix element (during the 1st algorithm run $n=1$ in the blocks 3 and 9); and $n$ is the number of matrix elements. After the algorithm run, the signal extreme point is displayed. According to the fact that the response is calculated in each segment of each MCTL section, the numbers of the conductor segment and the section (where the signal is localized) are known. The development of this approach resulted in creating an additional program module for TALGAT software [37]. Using this module, ordinary coupled lines were, first, studied and, then, compared with the results of CST MWS software. Then the real PCB bus of a spacecraft ANS was investigated under different conditions.

The computational complexity of the proposed approach involves multiple calculations of a signal waveform. There are as many calculations as segments of an MCTL section multiplied by the number of MCTL sections. We can define a signal waveform calculation with the complexity in Big O notation as $\mathrm{O}\left(\mathrm{n}^{2}\right)$. The complexity of the proposed approach is $\mathrm{O}\left(\mathrm{n}^{3}\right)$.

\section{Investigation of Coupled Transmission Lines}

\subsection{Meander Line with Two Turns}

The first structure to be studied was a meander line with two turns [30]. The circuit diagram and the cross section of this line are shown in Figure 9. The length of the line conductors is $27 \mathrm{~mm}$, the conductor width $(w)$ is $0.542 \mathrm{~mm}$, the conductor thickness $(t)$ is $0.1 \mathrm{~mm}$, the dielectric width $(d)$ is 5.149 $\mathrm{mm}$, the dielectric thickness $(h)$ is $0.3 \mathrm{~mm}$, the relative permittivity $\left(\varepsilon_{r}\right)$ is 4.5 , and the separation $(s)$ between the conductors is $0.217 \mathrm{~mm}$. The simulation was carried out without losses. Each conductor of the meander line was divided into 50 segments. Therefore, the voltage waveforms were calculated in 200 points along the whole length of the meander line (the MCTL section includes four conductors). The voltage maximum that is 1.14 times higher than the steady-state level was detected and located in segment 46 of the second conductor (shown by the arrow in Figure 9). The waveforms of the localized maximum in comparison with the ones found using CST WMS are presented in Figure 10. A good agreement of the obtained results is observed. The simulation in the CST MWS took $1080 \mathrm{~s}$. while the TALGAT software took $1 \mathrm{~s}$.

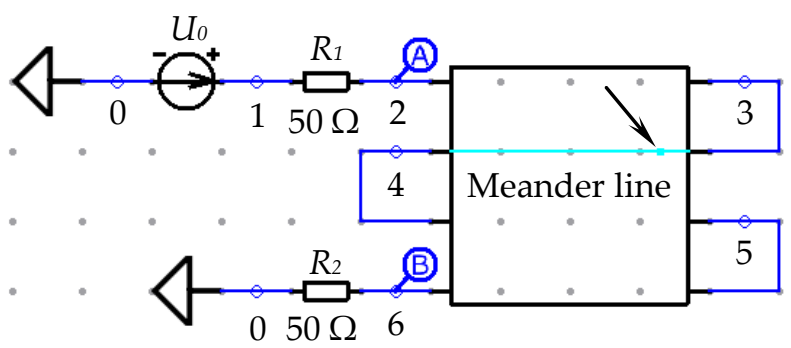

(a)

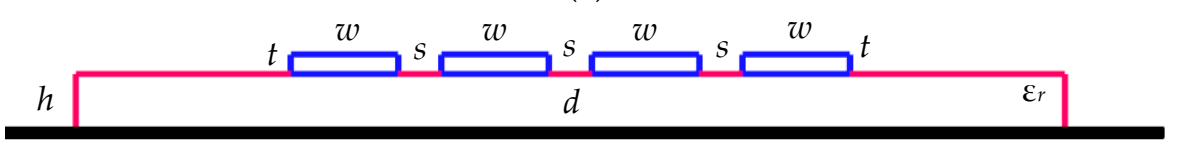

(b)

Figure 9. The circuit diagram (a) and the cross section (b) of the meander line with two turns. 


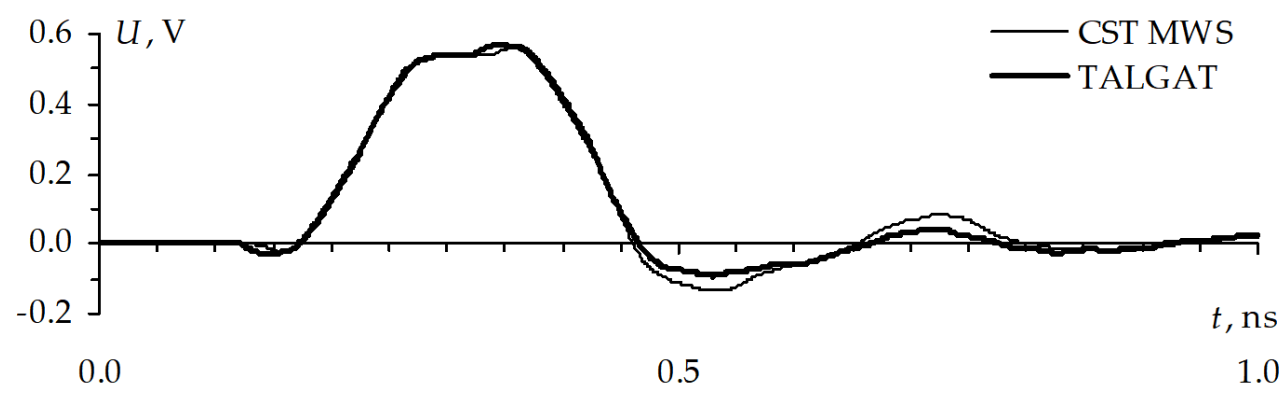

Figure 10. Comparison of the signal waveforms with the voltage maximum.

\subsection{Meander Line with Two Turns}

In order to understand the character of voltage transformation better, we investigated a simple meander line with one turn (cross section) [31]. The circuit diagram and the cross section of the line are shown in Figure 11.

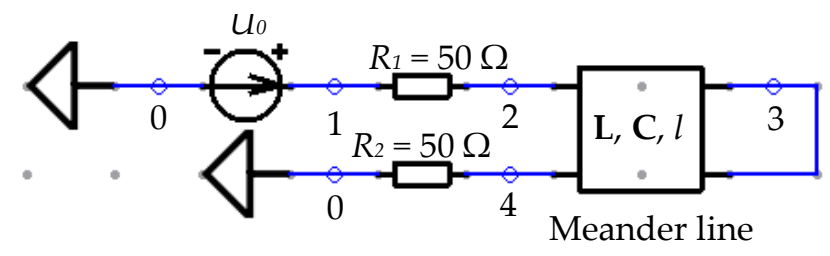

(a)

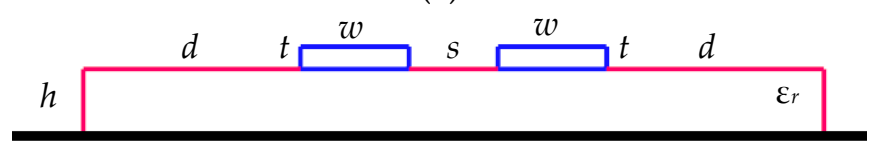

(b)

Figure 11. The circuit diagram (a) and the cross section (b) of the meander line with one turn.

The conductor width $(w)$ is $0.489 \mathrm{~mm}$, the conductor thickness $(t)$ is $0.1 \mathrm{~mm}, d=2 \cdot w$, the relative permittivity $\left(\varepsilon_{r}\right)$ is 4.5 , and the dielectric thickness $(h)$ is $0.3 \mathrm{~mm}$. The aim of the investigation was to calculate signal waveforms with extreme points at different separations ( $s$ ) (from $0.4 \mathrm{~mm}$ down to 1 $\mu \mathrm{m})$ between the conductors. The different separations were made in order to increase the mutual coupling between the line conductors. The most interesting results of this investigation are presented in Figure 12.

The highest voltage maximum $\left(U_{\max }\right)$ was localized with $s=8 \mu \mathrm{m}$ (Figure 12b). It is twice as high as the voltage amplitude at the input $\left(U_{2}\right)$. The comparison of the obtained result with CST MWS is shown in Figure 13. A good agreement of the obtained results is also observed. The simulation in the CST MWS took $840 \mathrm{~s}$ while the TALGAT software took $0.5 \mathrm{~s}$. 


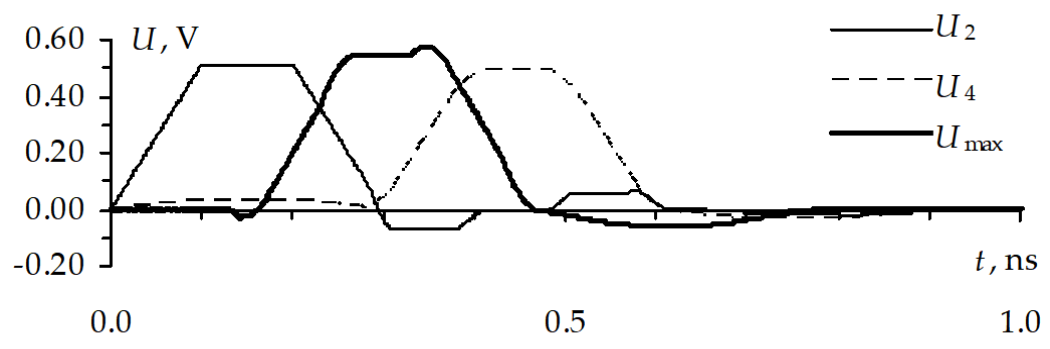

(a)

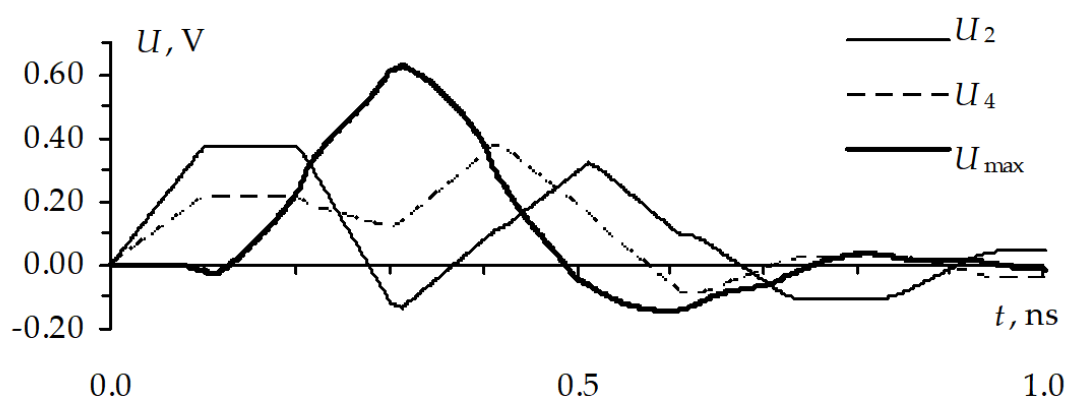

(b)

Figure 12. Voltage waveforms with $s=400 \mathrm{~mm}(\mathbf{a})$ and $8 \mu \mathrm{m}(\mathbf{b})$.

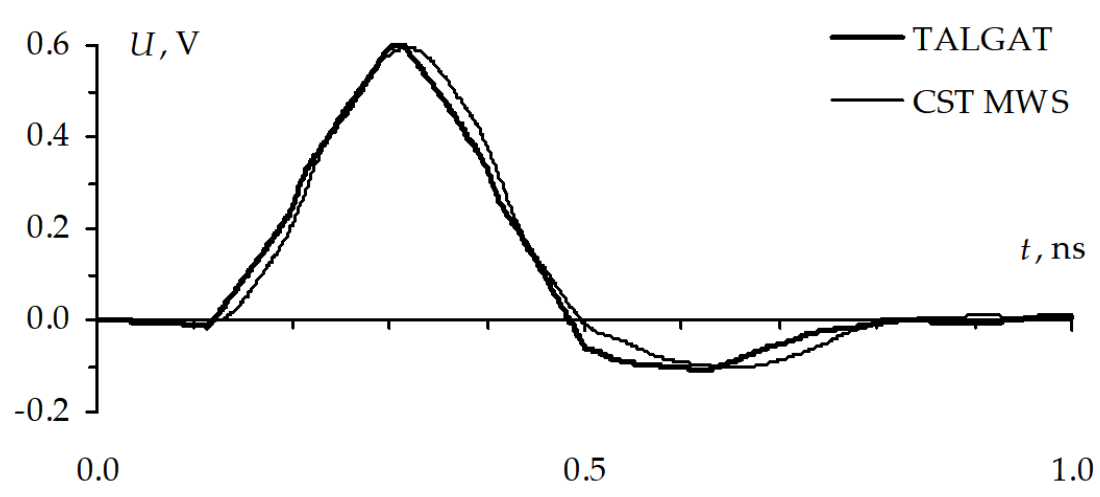

Figure 13. Comparison of the signal waveforms with the voltage maximum.

\section{Investigation of Spacecraft ANS PCB Bus}

After the study into the simple coupled transmission lines, a complex PCB bus of a spacecraft ANS was simulated then. The PCB bus fragment is shown in Figure 14, and the bus circuit diagram is shown in Figure 15. The resistors of $50 \mathrm{Ohm}$ are connected to the ends of each bus conductors. The conductor bend and via are approximately simulated as the capacitance of $1 \mathrm{pF}$ and inductance of 1 $\mathrm{nH}$, respectively. Each MCTL section cross section is modeled according to the PCB stack parameters. The losses were not taken into account. 


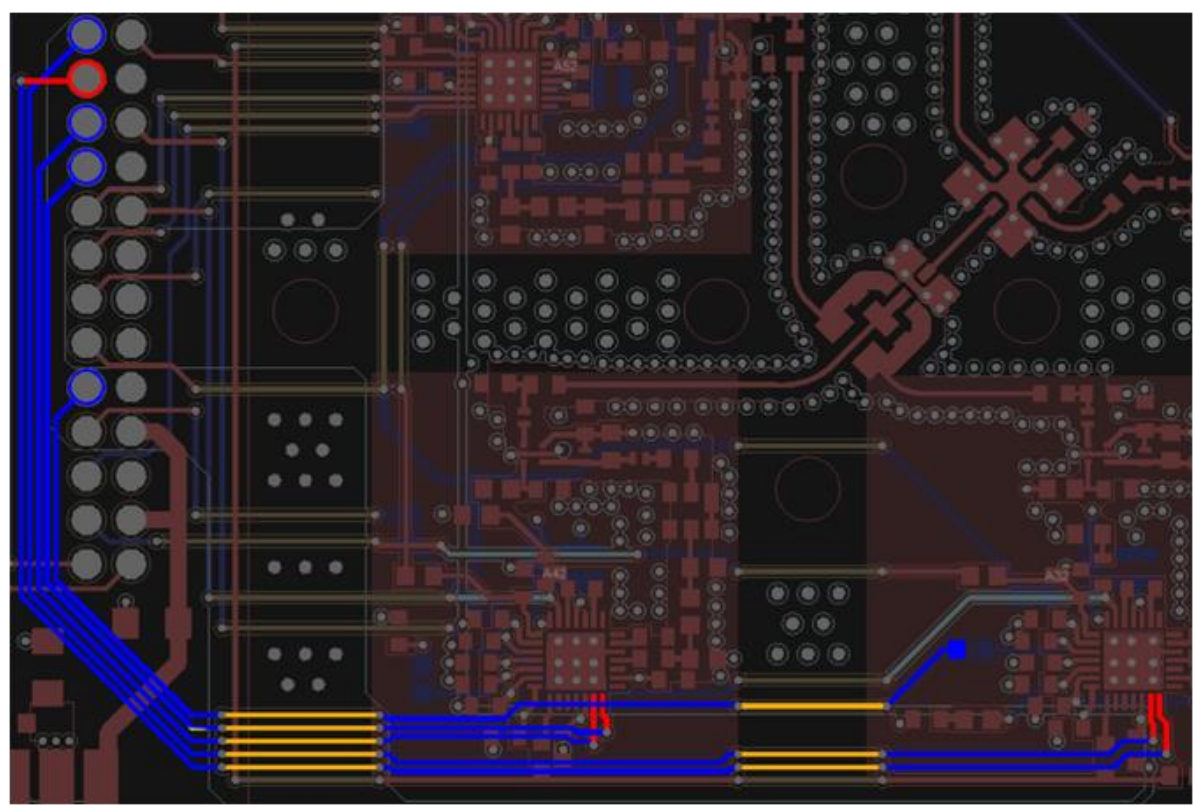

Figure 14. Investigated bus on printed circuit board (PCB) fragment.

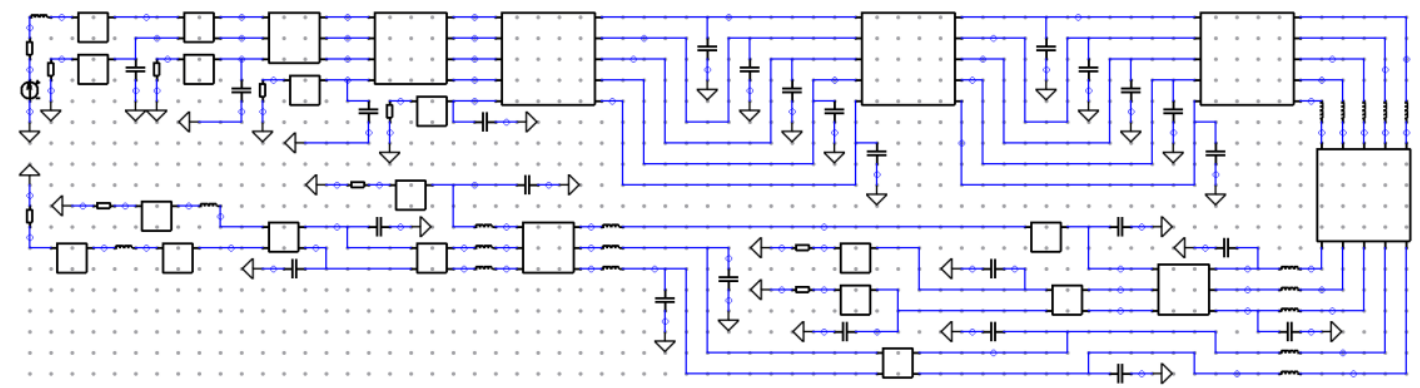

Figure 15. Circuit diagram of the PCB bus in TALGAT software.

\subsection{Influence of Ultrashort Pulse Duration on Localization of its Extreme Points in PCB Bus}

The investigation of the influence of ultrashort pulse duration on the localization of its extreme points in the PCB bus of a spacecraft ANS has been performed in [32]. This paper considers three durations of one ultrashort pulse, each with amplitude of $1 \mathrm{~V}$, as excitations. The waveforms of each pulse are presented in Figure 16. The first pulse $\left(U_{1}\right)$ has the rise, top, and fall times of $1 \mathrm{~ns}$, the second $\left(U_{2}\right)-100 \mathrm{ps}$, and the third $\left(U_{3}\right)-10 \mathrm{ps}$, and therefore the whole durations are $3,0.3$, and $0.03 \mathrm{~ns}$. Such choice of excitation parameters is determined by the fact that in such way not only useful signals, but interference ones, are considered.

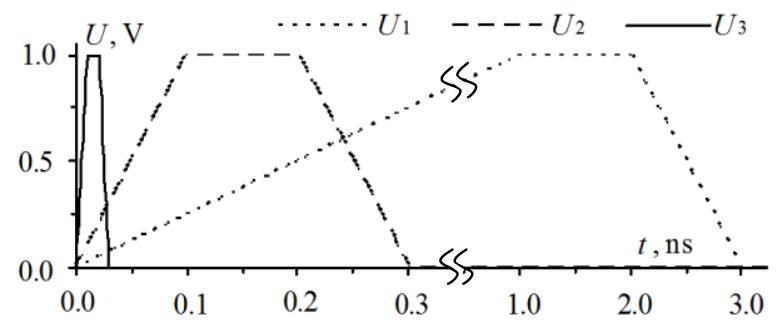

Figure 16. Excitation pulse waveforms with different durations.

We determine twenty voltage waveforms in each segment along each conductor of MCTL section, shown in Figure 15, but only the waveforms at the beginning $\left(U_{b}\right)$ and the end $\left(U_{e}\right)$ of the conductor, 
and the waveforms with the voltage maximum $\left(U_{\max }\right)$ and minimum $\left(U_{\min }\right)$ values, appearing under each excitation, are presented.

Let us consider the signal waveforms along the active conductor (Figure 17) and the locations of extreme points of these signals, which are shown by circles in Figure 18. We found that the signal maximum coincides with the signal waveform in the node under the excitation $U_{1}$, and minimums under excitations $U_{1}$ and $U_{2}$. By this reason the waveforms of these signals and their locations are not shown in Figures 17 and 18. Table 1 contains the values of the voltage peaks and the numbers of segments with their locations.

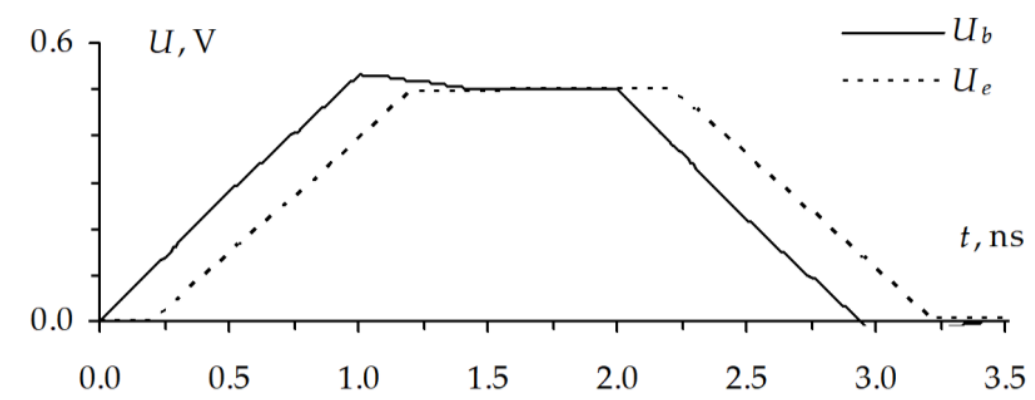

(a)

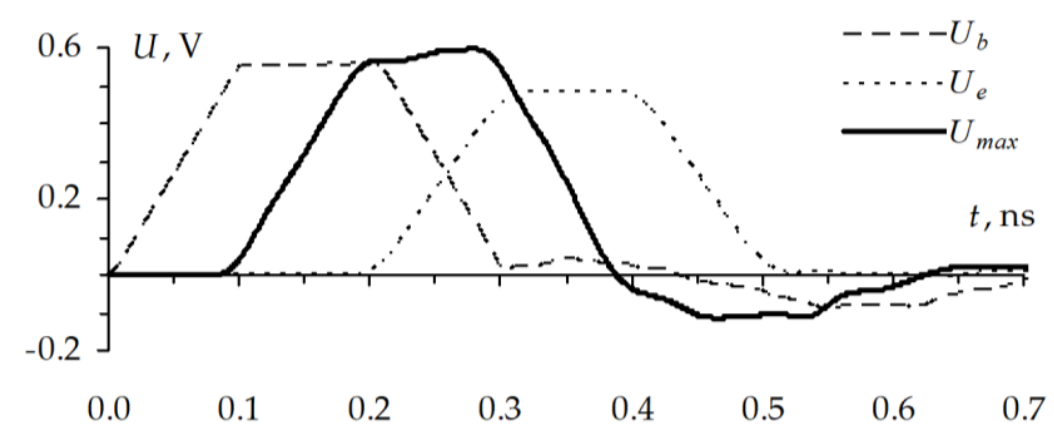

(b)

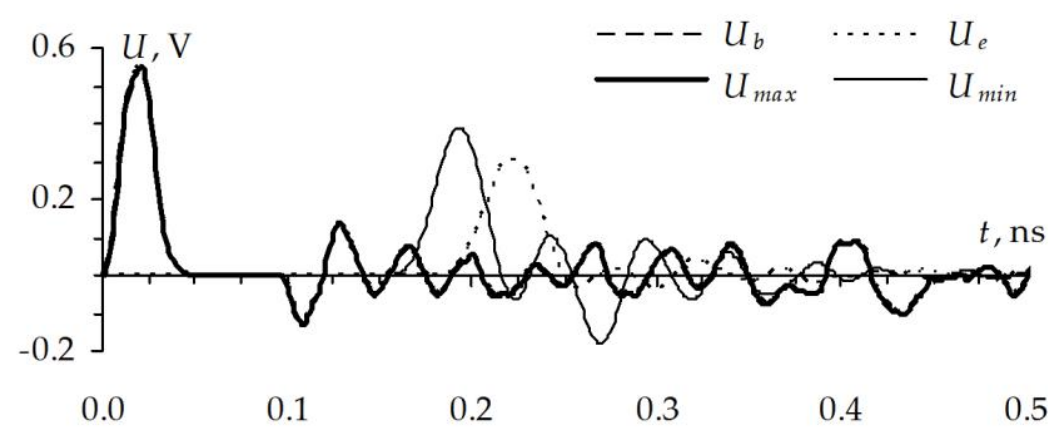

(c)

Figure 17. Signal waveforms obtained under the excitations $U_{1}(\mathbf{a}), U_{2}(\mathbf{b})$, and $U_{3}(\mathbf{c})$. 


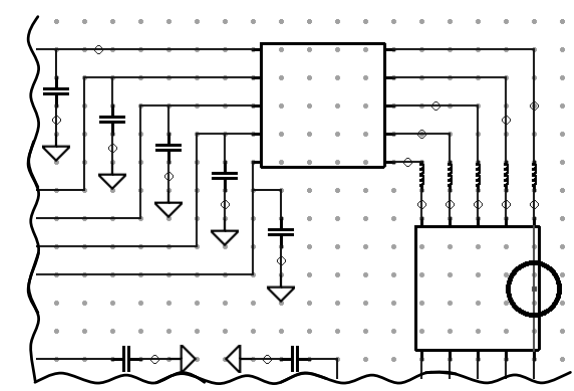

(a)

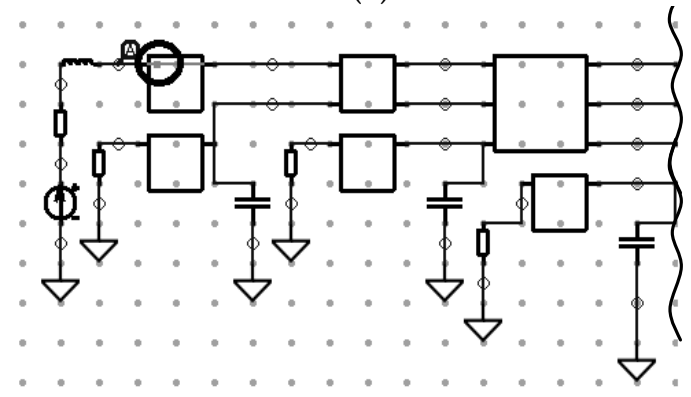

(b)

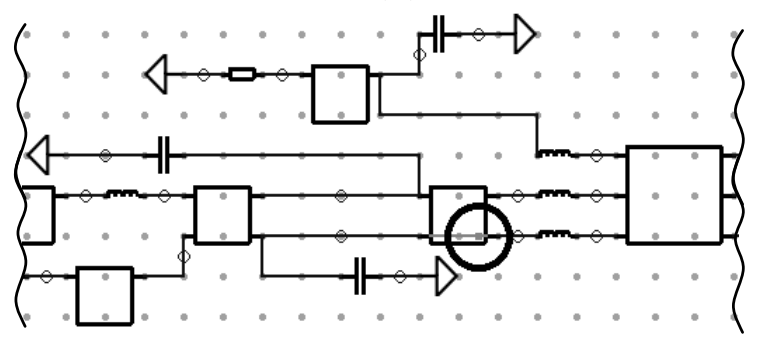

(c)

Figure 18. Location of maximums (a and $\mathbf{b}$ ) for signals from Figure $17 \mathrm{~b}, \mathrm{c}$, respectively, and also the location of minimum (c) for a signal from Figure 17c.

Table 1. Voltage extreme points and their localization parameters.

\begin{tabular}{cccccc}
\hline \multirow{2}{*}{ Excitation } & \multirow{2}{*}{ Figure } & \multicolumn{2}{c}{$U_{\max }$} & \multicolumn{2}{c}{$U_{\min }$} \\
\cline { 3 - 6 } & & Voltage, $\mathbf{V}$ & Segment, Figure & Voltage, $\mathbf{V}$ & Segment, Figure \\
\hline$U_{1}$ & $17 \mathrm{a}$ & 0.530 & 1 & -0.05 & 1 \\
$U_{2}$ & $17 \mathrm{~b}$ & 0.597 & $10(18 \mathrm{a})$ & -0.11 & 20 \\
$U_{3}$ & $17 \mathrm{c}$ & 0.552 & $5(18 \mathrm{~b})$ & -0.18 & $3(18 \mathrm{c})$ \\
\hline
\end{tabular}

Let us consider the excitation $\left(U_{1}\right)$. The extreme points of the waveforms presented in Figure 17a and their location coincide with the waveforms at the conductor's ends. Let us consider the $U_{2}$ and $U_{3}$ excitations which can be relegated to high-speed or interfering signals because their durations are shorter. Under the $U_{2}$ excitation, voltage maximum is $0.59 \mathrm{~V}$ (Figure $17 \mathrm{~b}$ ) which is $18 \%$ higher than the steady-state level of $0.5 \mathrm{~V}$. The maximum is localized in segment 10 (Table I) in one of the transmission line sections with five conductors that are located on another layer (Figure 18a). Under the $U_{3}$ excitation, not only the voltage maximum of $0.552 \mathrm{~V}$ that exceeds the $0.5 \mathrm{~V}$ level by $10 \%$ is detected, but also a minimum of minus $0.18 \mathrm{~V}$ (Figure $17 \mathrm{c}$ ) or minus $36 \%$ of $0.5 \mathrm{~V}$, which is lower than the level of zero. Moreover, it is shown in Table 1 and Figure $18 b, c$ that the extreme points are localized in absolutely different places.

Let us consider the results for the $U_{3}$ excitation. Besides the appearance of peak values, it was found that there appeared multiple reflections and the signal amplitude at the output of the line 
decreased. It was $0.4 \mathrm{~V}$ in Figure $17 \mathrm{c}$, which is $20 \%$ lower than the $0.5 \mathrm{~V}$ level. The investigation shows specific aspects of detecting and localizing extreme points of the ultrashort pulse with various durations. For example, the highest maximum value (about $0.6 \mathrm{~V}$ ) is detected under the $U_{2}$ excitation as we can see from Table 1 . The extreme points are located in different transmission line sections and segments along these conductors (i.e., in different places of the PCB).

\subsection{Influence of Ultrashort Pulse Duration on Localization of Crosstalk Extreme Points in PCB Bus}

The investigation of the influence of ultrashort pulse duration on the localization of crosstalk extreme points in a PCB bus of a spacecraft ANS has been performed by [33]. This study used four generators and conductor 3 (central) was passive (Figure 19). In this figure, the arrows show the generators, and the conductors are numbered. The same durations of the ultrashort pulse (shown in Figure 16) were used as excitations. The simulation results for conductor 3 (central) are shown in Figure 20 and the segments with peak voltage values are pointed in Figure 21.

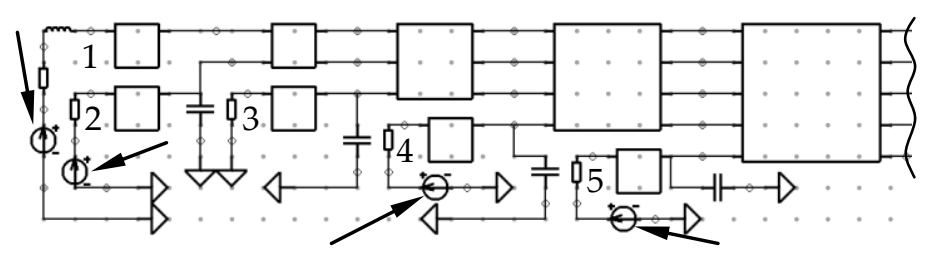

Figure 19. Diagram fragment with four generators.

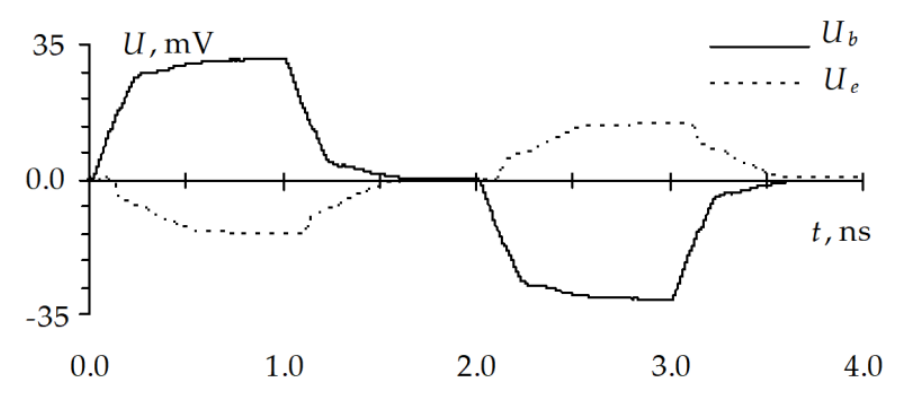

(a)

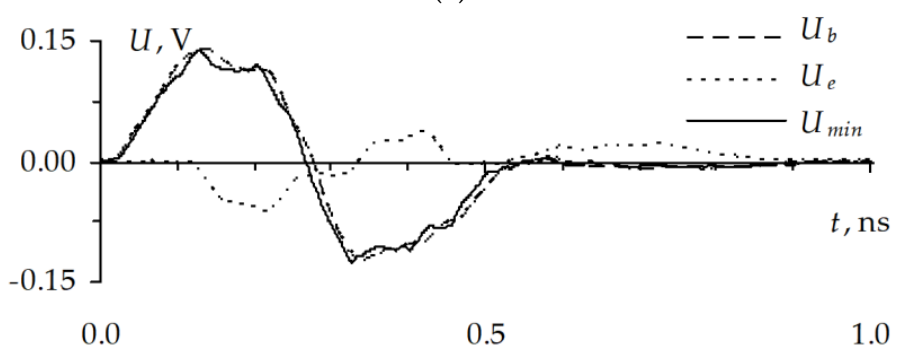

(b)

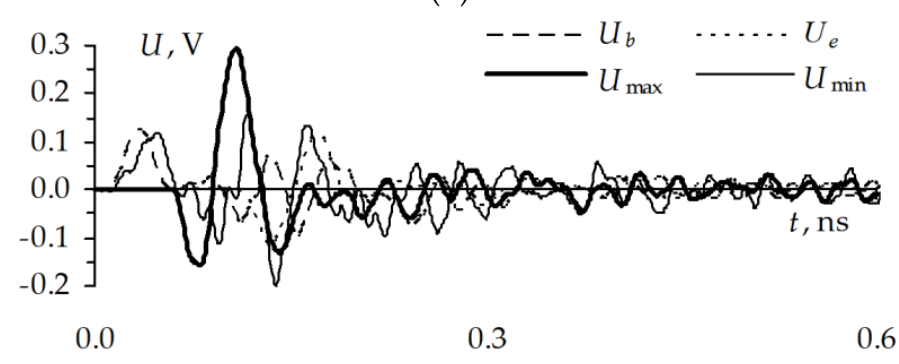

(c)

Figure 20. Waveforms along conductor 3 under the excitations $U_{1}(\mathbf{a}), U_{2}(\mathbf{b})$, and $U_{3}(\mathbf{c})$. 


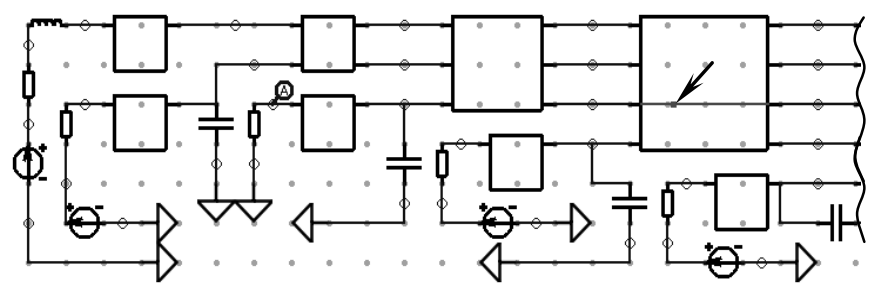

(a)

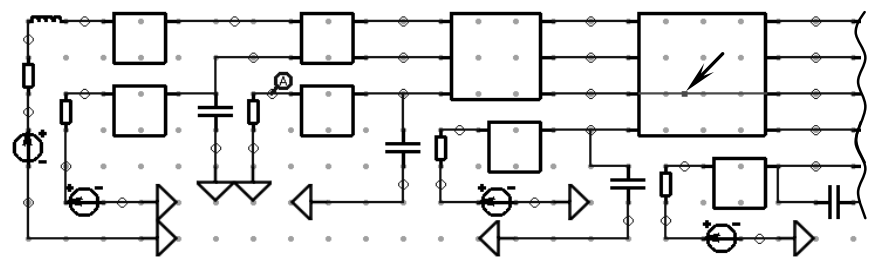

(b)

Figure 21. Maximum locations for signals from Figure 20b (a) and иFigure 20c (b).

The voltage extreme points and its location segment numbers are shown in Table 2. Consider the excitation of the useful signal $\left(U_{1}\right)$. The signal waveforms calculated for conductor 3 are presented in Figure 20a, but their extremes and location are similar to the waveforms calculated at the ends of the conductor. Let us consider the excitations $U_{2}$ and $U_{3}$ which can be regarded as interference signals. These excitations have the shorter durations than the useful ones. Some voltage maximums are detected, but they are located in the diagram nodes. The voltage minimum calculated under the excitation $U_{2}$ is minus $0.126 \mathrm{~V}(25.2 \%$ of $0.5 \mathrm{~V})$. The location of this extreme point is in the segment 5 . But the voltage minimum calculated under the excitation $U_{3}$ is minus $0.199 \mathrm{~V}(39.8 \%$ of $0.5 \mathrm{~V})$ and is located in segment 8 .

Table 2. Crosstalk extreme points and their localization parameters.

\begin{tabular}{cccccc}
\hline \multirow{2}{*}{ Excitation } & \multirow{2}{*}{ Figure } & \multicolumn{2}{c}{$U_{\max }$} & \multicolumn{2}{c}{$U_{\min }$} \\
\cline { 3 - 6 } & & Voltage, $\mathbf{V}$ & Segment, Figure & Voltage, V & Segment, Figure \\
\hline$U_{1}$ & $20 \mathrm{a}$ & 0.031 & 1 & -0.031 & 1 \\
$U_{2}$ & $20 \mathrm{~b}$ & 0.139 & 1 & -0.126 & $5(21 \mathrm{a})$ \\
$U_{3}$ & $20 \mathrm{c}$ & 0.292 & 1 & -0.199 & $8(21 \mathrm{~b})$ \\
\hline
\end{tabular}

This study unveils the specific details of detecting and localizing extreme points for an ultrashort pulse with different durations. The highest maximum value $(0.145 \mathrm{~V}, 29 \%$ of $0.5 \mathrm{~V})$ appears under the excitation $U_{3}$ as it shown in Table 1 (the full duration of the pulse was $0.03 \mathrm{~ns}$ ). The highest minimum value (minus $0.199 \mathrm{~V}, 39.8 \%$ of $0.5 \mathrm{~V}$ ) is also under the excitation $U_{3}$. Considering that the maximum acceptable crosstalk level in the ANS PCB bus should be less than $10 \%$ of signal amplitude in the active conductor, it follows that all detected extreme points (excluding those under the excitation $U_{1}$ ) dissatisfy this condition.

\subsection{Simulation of ESD Effects on PCB Bus}

The simulation of ESD effects on a PCB bus of a spacecraft ANS have been described in [38]. The ESD, whose parameters and selection justification are described in [39], was chosen as excitation. The current waveform according to the IEC 61000-4-2 [40] standard was used. It was excited on each conductor in turns and the voltage waveforms were calculated along each conductor. In addition, the case was considered when all conductors, except the central one, were under the ESD excitation. The results of the last case are presented further. The simulation results for the central conductor when the four conductors were active and the central one was passive are presented in Figure 22. 


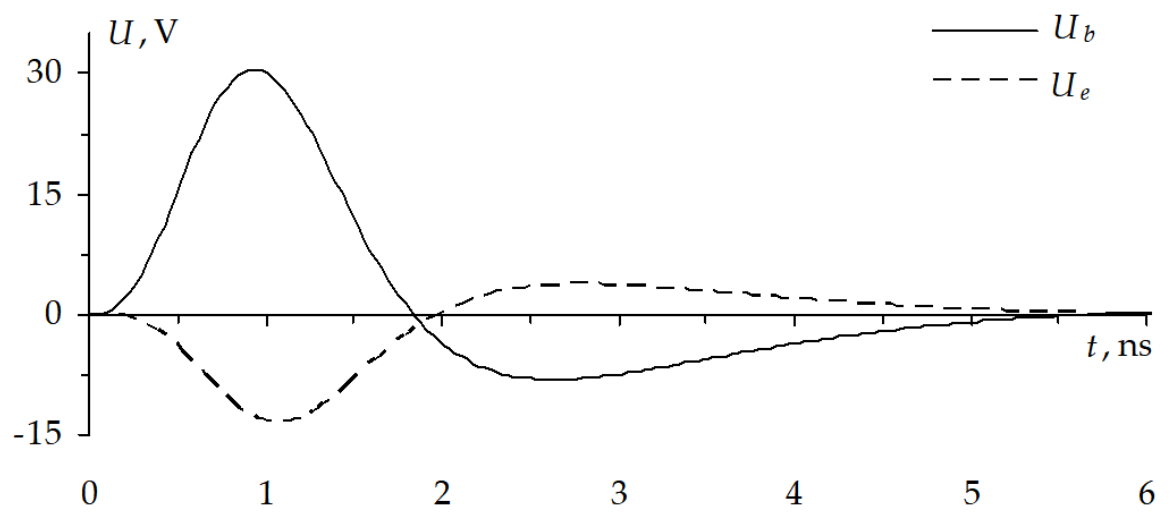

Figure 22. Waveforms along conductor 3 with active conductors 1, 2, 4, and 5 .

The waveforms calculated at the beginning of one of the active conductors are shown in Figure 23, (waveforms for others are almost the same).

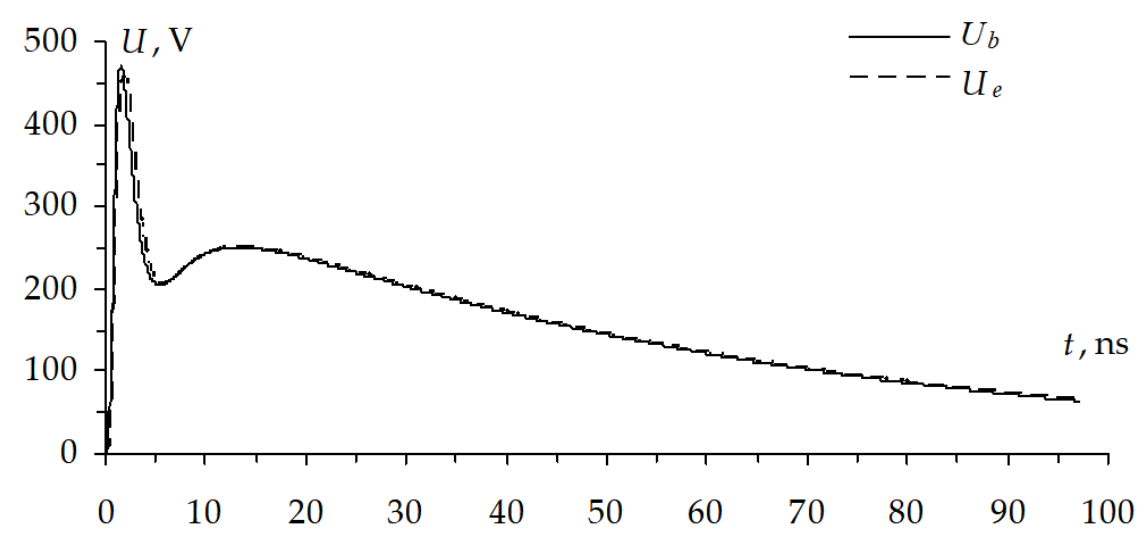

Figure 23. Waveforms along conductor 1 with active conductors 1, 2, 4, and 5 .

Let us consider the signal waveforms in the active conductor 1 . The voltage amplitude of 470 $\mathrm{V}$, which is rather dangerous for integrated circuits (IC), is observed in this conductor (Figure 23). The extreme points coincide with the voltages at the input or the end of the conductor, and therefore they are not shown in the figures. Let us consider the signal waveforms in the passive conductor (Figure 22). The crosstalk amplitude is $32 \mathrm{~V}$ which is $6.8 \%$ of the signal level in the active conductors. The significant negative voltage (minus $14 \mathrm{~V}$ ) is also observed.

This work demonstrates the importance of studying the ANS PCB bus under ESD excitation, and therefore the simulation of ESD excitation on several bus conductors at once has also been carried out. First of all, it showed that considerable peak voltages along the investigated bus conductors (higher than the voltages at the ends of the conductors) did not appear under the ESD excitation (in contrast to the ultrashort pulse $[32,34,41])$.

Another important finding was that the voltage amplitude in the active conductor can be near 0.5 $\mathrm{kV}$ and could disable an integrated circuit (IC). The crosstalk can also be dangerous because it can be deemed in many circuits as the useful signal (1-2 ns, $32 \mathrm{~V})$ and become the reason for IC upset. Moreover, we should note that these results were obtained for the resistances of $50 \mathrm{Ohm}$ while the high input impedance of a real IC can double these values. Such amplitudes can strongly influence the operation of radio-electronic equipment of critical significance.

\section{Optimization by Genetic Algorithms}

In the following investigations, we have used the optimization by genetic algorithms in various options. 


\subsection{Optimization of Ultrashort Pulse Duration by Criteria of Peak Voltage Maximization in PCB Bus}

The simple binary-coded GA (a number of bits for each parameter is taken 16 as default in TALGAT software) was launched with the following parameters [34]: coefficient of mutation was 0.1 and coefficient of crossover was 0.5 . The rise, flat top, and fall durations were separately optimized so the total duration range was from $1 \mathrm{~ns}$ to $10 \mathrm{ps}$ (as shown in Figure 16), the number of chromosomes was three and five, and the number of populations was five, 25, 50, and 75 . The sum of peak voltages from the ends of the PCB bus conductors 1, 3, and 5 was maximized (these points are considered as examples of the critical places where the bus conductors are connected to the other PCB components). The aim of the second part of the investigation was to define the rise, flat top, and fall duration values of the ultrashort pulse, at which the sum of voltages in the preset points will be maximal.

The results of the GA run, including the calculation of time, $(t)$, for the rise, flat top and fall times of the ultrashort pulse are presented in Table 3 (the number of chromosomes is five). The GA was launched five times for each combination of the population number $\left(N_{P}\right)$. This is caused by the necessity to check the convergence of the fitness function results. The diagram of convergence of the $U_{\max }$ values with a different number $(n)$ of fitness function (product of the number of chromosomes and the number of populations) calculations is shown in Figure 24. A total of 20 voltage waveforms were calculated in each segment along each conductor of each MCTL section from Figure 15 with the obtained results for the highest fitness function value (run 2 from Table 3, when the number of populations was 75), but only the waveforms at the beginning $\left(U_{b}\right)$ and end $\left(U_{e}\right)$ of the conductor and also the waveforms with the voltage maximum $\left(U_{\max }\right)$ values are presented. The results are presented only for one active and one passive conductor with the highest crosstalk amplitude.

Table 3. Results of five genetic algorithm (GA) runs for five chromosomes.

\begin{tabular}{ccccccc}
\hline$N_{\boldsymbol{P}}$ & Number of a Run & $\boldsymbol{t}, \mathbf{s}$ & $\boldsymbol{t}_{\boldsymbol{r}}, \mathbf{n s}$ & $\boldsymbol{t}_{\boldsymbol{d}}, \mathbf{n s}$ & $\boldsymbol{t}_{\boldsymbol{f}}, \mathbf{n s}$ & $\boldsymbol{U}_{\text {max }}, \mathbf{V}$ \\
\hline \multirow{3}{*}{5} & 1 & 441.407 & 0.989 & 0.0114 & 0.800 & 0.551147 \\
& 2 & 452.437 & 0.500 & 0.0199 & 0.879 & 0.545629 \\
& 3 & 486.692 & 0.447 & 0.0356 & 0.582 & 0.534152 \\
& 4 & 486.508 & 0.707 & 0.0251 & 0.127 & 0.531801 \\
& 5 & 510.564 & 0.111 & 0.0550 & 0.954 & 0.524602 \\
\hline \multirow{3}{*}{10} & 1 & 983.870 & 0.663 & 0.0119 & 0.925 & 0.550284 \\
& 2 & 1045.06 & 0.487 & 0.0100 & 0.772 & 0.551331 \\
& 3 & 1133.29 & 0.429 & 0.0102 & 0.652 & 0.549775 \\
& 4 & 1204.64 & 0.894 & 0.0111 & 0.923 & 0.550668 \\
& 5 & 1280.82 & 0.951 & 0.0184 & 0.719 & 0.547373 \\
\hline & 1 & 3679.80 & 0.9160 & 0.0108 & 0.874 & 0.551185 \\
& 2 & 3689.23 & 0.3980 & 0.0114 & 0.938 & 0.551207 \\
& 3 & 3686.37 & 0.7300 & 0.0103 & 0.761 & 0.551353 \\
& 4 & 3649.92 & 0.0135 & 0.0104 & 0.820 & 0.553568 \\
& 5 & 3673.96 & 0.6580 & 0.0103 & 0.789 & 0.551315 \\
\hline
\end{tabular}




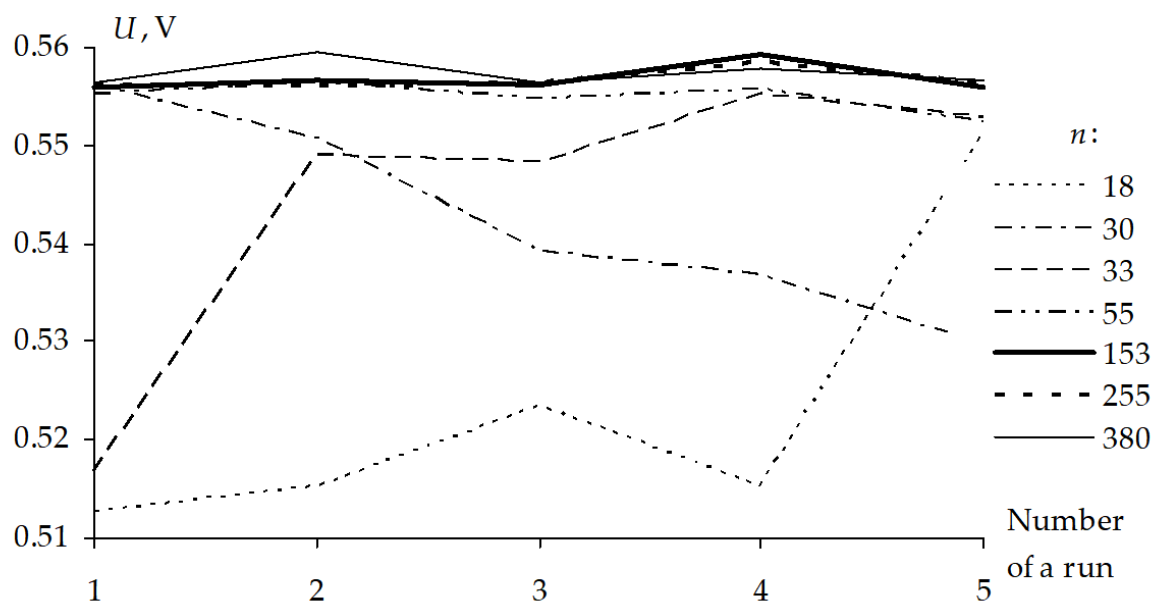

Figure 24. $U_{\max }$ values for five GA runs with different $n$.

The voltage waveforms calculated along the active conductor are presented in Figure 25a, and the location of the ultrashort pulse maximum is shown in Figure 25b. The voltage waveforms along the passive conductor are shown in Figure 26a, and the crosstalk maximum location is shown in Figure 26b. Let us consider the optimization results. With the maximal number of calculations, the fitness function value is $0.55459 \mathrm{~V}$ (Table 3) and the average (of five runs) calculation time is $5767.5 \mathrm{~s}$. The fitness function results show a good convergence when the numbers of calculations are 255 and 380, the results differ only in the third decimal place. The voltage maximum in the active conductor is detected and localized with the optimized parameters (short rise and fall times, and maximal flat top duration) which are obtained at run 2 (the number of chromosomes is five and the number of populations is 75) from Table 3. The detected voltage maximum is $0.68 \mathrm{~V}$ (Figure 25a) which is $36 \%$ higher than the steady state level. The maximum is located in segment 6 (Figure 25b) in an MCTL section of another layer of the PCB.

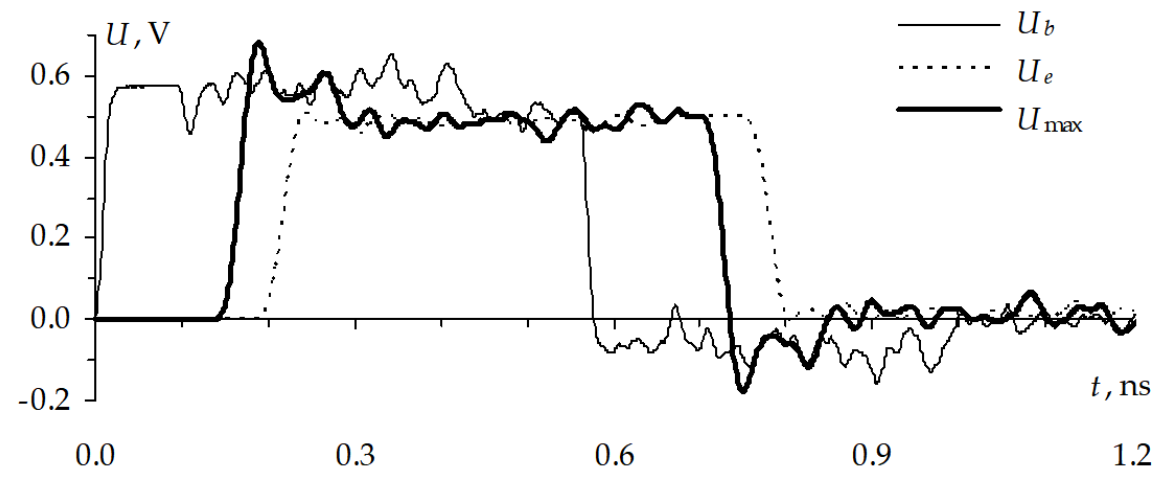

(a)

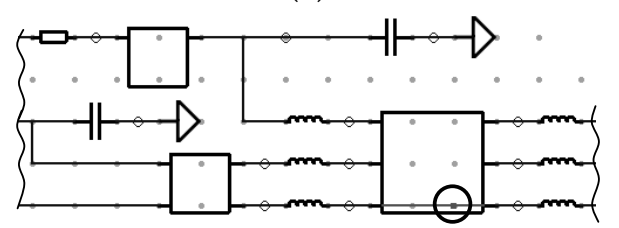

(b)

Figure 25. Voltage waveforms along the active conductor (a) and the voltage maximum location (b). 


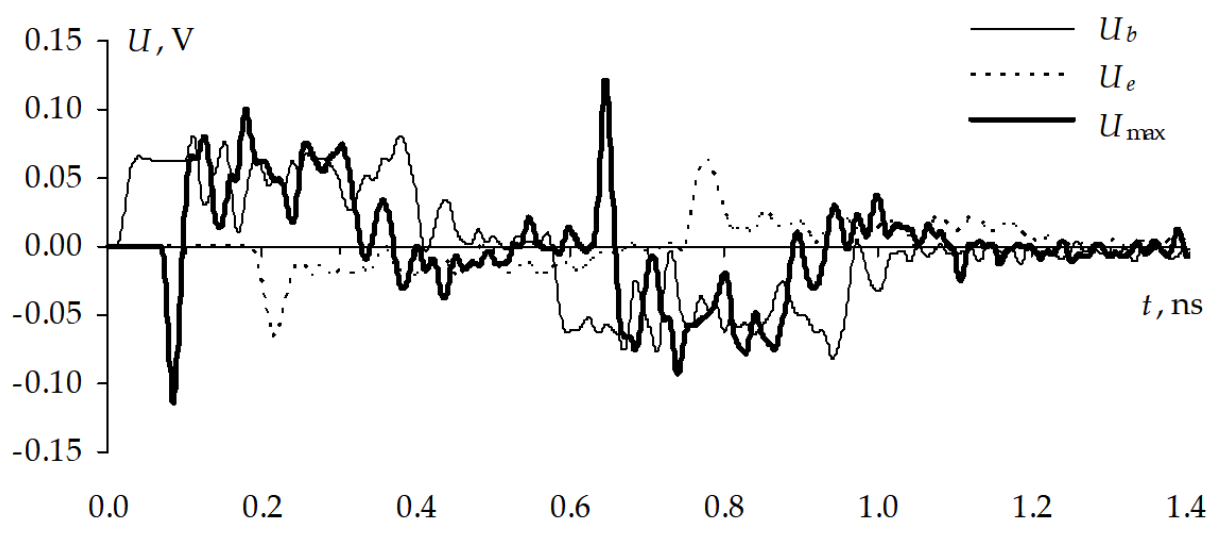

(a)

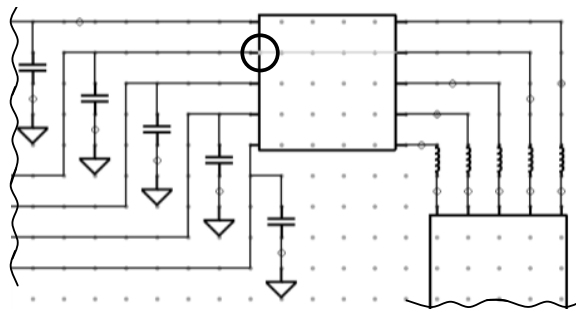

(b)

Figure 26. Voltage waveforms along the passive conductor (a) and the crosstalk maximum location (b).

Moreover, the crosstalk maximum is detected and localized in segment 1 in one of the five conductor transmission line sections. The crosstalk maximum is $0.12 \mathrm{~V}$ which is $24 \%$ of a steady-state level. The investigation shows the importance of the GA for optimization for detecting and localizing signal peak values or the sum of several signals under excitation of the ultrashort pulse with different durations.

\subsection{Optimization of Ultrashort Pulse Duration by Criteria of Peak Voltage Maximization in PCB Bus}

The investigation focused on the influence of crossover and mutation coefficients on the GA for optimization of the ultrashort pulse duration by criteria of peak voltage maximization in the PCB bus [35] and employed a simple binary-coded GA. The investigation consisted of two parts. In the first part, the mutation coefficient $\left(k_{m}\right)$ ranged from 0.01 up to 0.08 , and, in the second part, the crossover coefficient $\left(k_{c}\right)$ ranged from 0.1 up to 0.8 . In the first part, $k_{c}=0.5$ and, in the second part $k_{m}=0.1$. Three parameters, $t_{r}, t_{d}$, and $t_{f}$, were optimized in the range from $1 \mathrm{~ns}$ down to $0.01 \mathrm{~ns}$ when the chromosome number was five and the number of populations was 26 (so the total GA calculation number was 130). The sum of peak voltages at the ends of the PCB bus conductors 1,3 , and 5 was maximized. The aim of the optimization was to define the rise, flat top, and fall duration values of the ultrashort pulse, at which the sum of voltages $\left(U_{\mathrm{SUM}}\right)$ in the preset points will be maximal. It is important to notice that it is necessary to choose a greater number of GA calculations to provide a more complete investigation. The simulation of excitation by several sources is also useful, but due to the fact that the work presents the preliminary stage of the investigation only, it was decided to choose a small number of calculations and one source only. The values of the sum of maximum voltages in the preset points for the first part of the investigation are presented in Table 4 . The presented results are obtained with $k_{c}=0.1$ and different $k_{m}$ for ten GA runs. 
Table 4. Values of $U_{\text {sum }}$ and $\mathrm{V}$ with different $k_{m}$ for ten GA runs.

\begin{tabular}{ccccc}
\hline \multirow{2}{*}{ Run } & \multicolumn{3}{c}{$\boldsymbol{k}_{\boldsymbol{m}}$} \\
\cline { 2 - 5 } & $\mathbf{0 . 0 1}$ & $\mathbf{0 . 0 3}$ & $\mathbf{0 . 0 5}$ & $\mathbf{0 . 0 8}$ \\
\hline 1 & 0.50819 & 0.55159 & 0.55108 & 0.54131 \\
2 & 0.51607 & 0.55122 & 0.55196 & 0.55146 \\
3 & 0.54913 & 0.55704 & 0.55005 & 0.54974 \\
4 & 0.55094 & 0.55081 & 0.55100 & 0.55503 \\
5 & 0.50799 & 0.55284 & 0.55043 & 0.55127 \\
6 & 0.53153 & 0.54920 & 0.55317 & 0.55092 \\
7 & 0.52685 & 0.55092 & 0.55079 & 0.54232 \\
8 & 0.53983 & 0.55271 & 0.55272 & 0.55108 \\
9 & 0.54009 & 0.55110 & 0.55128 & 0.54909 \\
10 & 0.54309 & 0.55132 & 0.55120 & 0.55041 \\
\hline
\end{tabular}

The convergence diagrams for the best fitness function results obtained by different runs are shown in Figure $27 \mathrm{a}$, where $N_{\mathrm{C}}$ is the calculation number and $N_{\mathrm{R}}$ is the run number. The presented results are obtained when $k_{m}=0.03$ because with such $k_{m}$ the $U_{\max }$ in Table 4 is the highest. Convergence diagrams of the arithmetic average of ten runs with different $k_{m}$ are shown in Figure 27b. The GA run results of the second part of the investigation (the sum of maximum voltages at I, II, and III points when $k_{m}=0.1$ and $k_{c}$ is different) are presented in Table 4 .

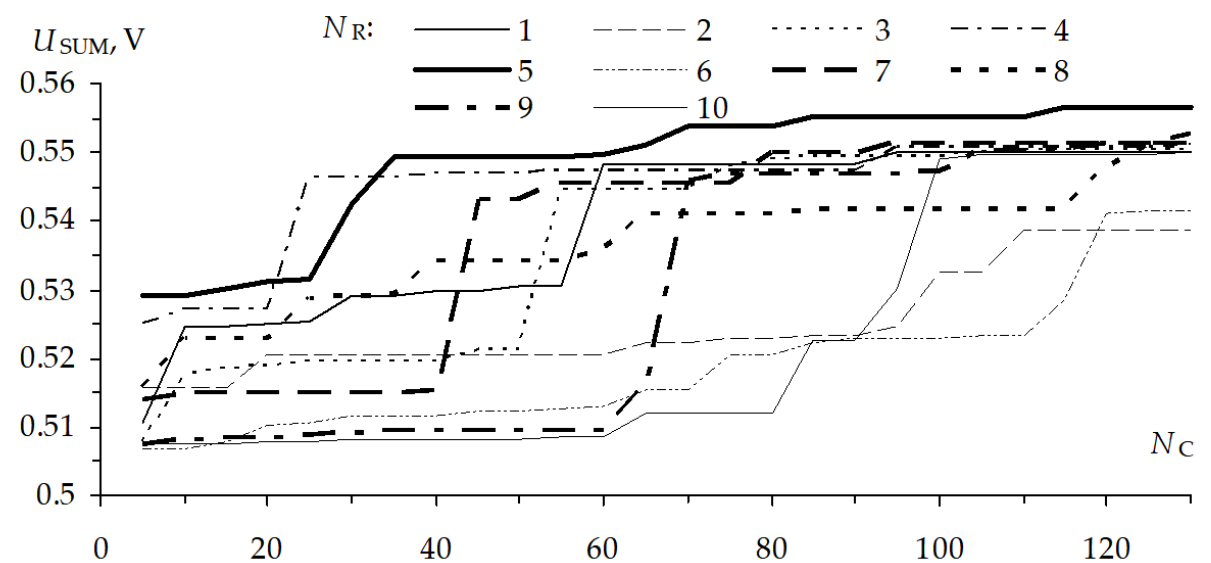

(a)

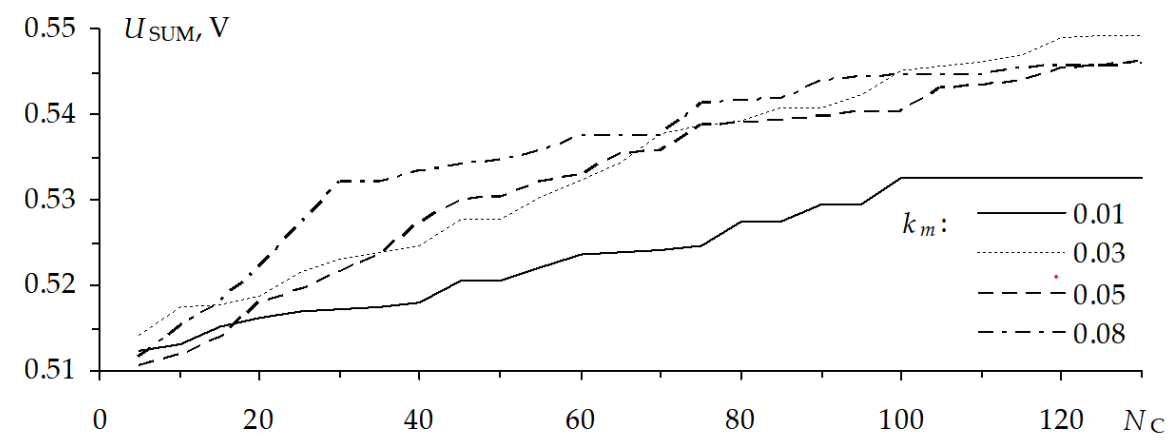

(b)

Figure 27. Convergence diagrams of the fitness function values for each run with $k_{m}=0.03$ (a) and the arithmetic average of runs with different $k_{m}(\mathbf{b})$.

The convergence diagrams for the best fitness function results obtained at different runs are shown in Figure 28a. The presented results are obtained when $k_{c}$ table $=0.8$ because with such $k_{c}$ the $U_{\max }$ in Table 3 is the highest. The convergence diagrams of the arithmetic average of ten runs with different $k_{c}$ 
are shown in Figure 28b. As regards the best fitness function result, all obtained values are similar and differ in the third decimal place. Additionally, the highest result $(0.55704 \mathrm{~V})$ is obtained with $k_{\mathrm{m}}=0.03$ (Table 4). Let us consider the results of the second part of the investigation. As regards the best fitness function result, the situation is the same as for the first part of the investigation, all obtained results are similar and differ in the third decimal place only. Meanwhile, the highest result is $0.55448 \mathrm{~V}$ (obtained with $k_{c}=0.8$ ).

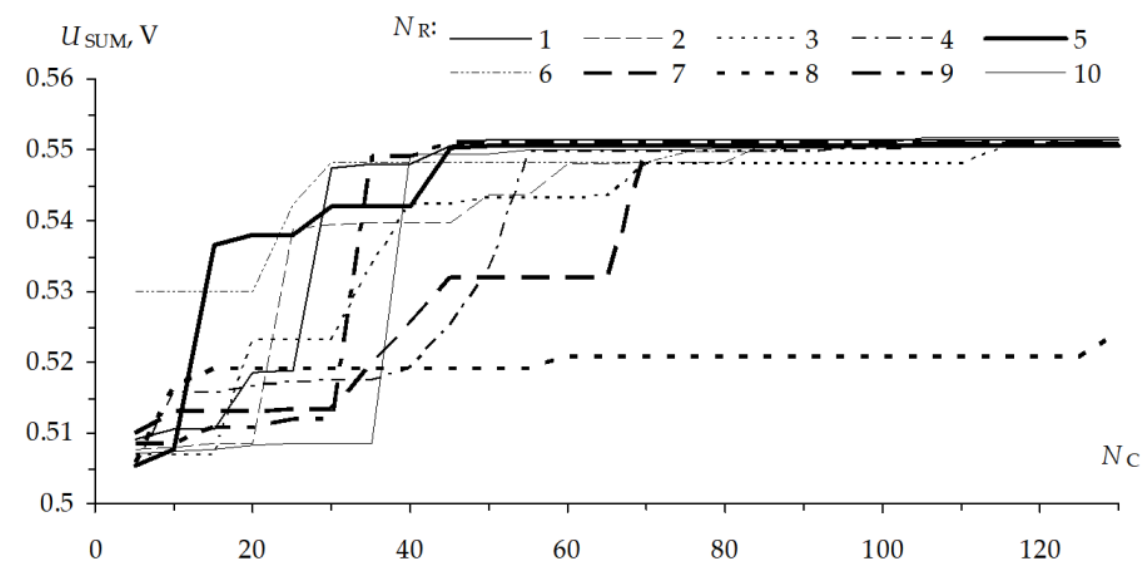

(a)

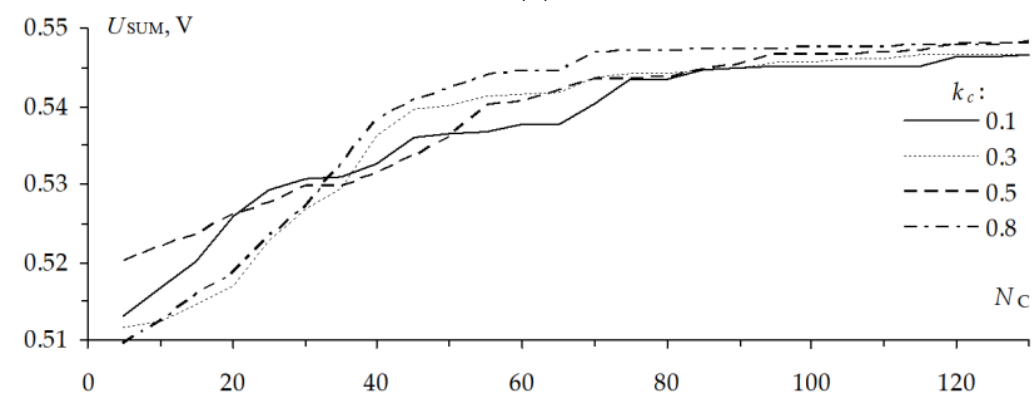

(b)

Figure 28. Convergence diagrams of the fitness function values for each run with $k_{c}=0.8(\mathbf{a})$ and the arithmetic average of runs with different $k_{c}(\mathbf{b})$.

The investigation shows the importance of the GA for optimization of the sum of several signals under excitation of the ultrashort pulse with different durations. For instance, as we can see from Tables 4 and 5, when we change either mutation or crossover coefficients, the highest fitness function is near $0.55 \mathrm{~V}$ and $0.55132 \mathrm{~V}$ in Table 4, and $0.55173 \mathrm{~V}$ in Table 5. It is found that the mutation coefficient variation strongly influences the convergence of results. Indeed, changing the mutation coefficient results in convergence deceleration and the diagrams are observed to stand out. The crossover coefficient variation gives us the fastest convergence (with $k_{c}=0.8$ it is at the 70th calculation). However, these coefficient variations hardly influence the detected peak voltages of the ultrashort pulse and crosstalk. The obtained peak voltages have the same amplitudes. 
Table 5. $U_{\text {sum }}$ and $V$ values with different $k_{\mathcal{c}}$ for ten GA runs.

\begin{tabular}{ccccc}
\hline \multirow{2}{*}{ Run } & \multicolumn{3}{c}{$\boldsymbol{k}_{\boldsymbol{c}}$} \\
\cline { 2 - 5 } & $\mathbf{0 . 1}$ & $\mathbf{0 . 3}$ & $\mathbf{0 . 5}$ & $\mathbf{0 . 8}$ \\
\hline 1 & 0.55444 & 0.55214 & 0.55166 & 0.55072 \\
2 & 0.53083 & 0.55013 & 0.55177 & 0.55128 \\
3 & 0.55169 & 0.54860 & 0.55027 & 0.55167 \\
4 & 0.55041 & 0.54996 & 0.55092 & 0.52187 \\
5 & 0.55279 & 0.54988 & 0.55121 & 0.55254 \\
6 & 0.55102 & 0.55165 & 0.55180 & 0.55118 \\
7 & 0.55150 & 0.55182 & 0.54766 & 0.55448 \\
8 & 0.55120 & 0.55060 & 0.55040 & 0.55024 \\
9 & 0.55191 & 0.55052 & 0.55122 & 0.55142 \\
10 & 0.55058 & 0.55116 & 0.55173 & 0.55012 \\
\hline
\end{tabular}

\subsection{Optimization of PCB Bus Loads by Criteria of Peak Voltage Minimization}

The investigation involved a GA for optimization of the PCB bus loads by criteria of peak voltage minimization [41]. The GA with binary encoding was launched with the following parameters: the coefficient of mutation $=0.1$ and the coefficient of crossover $=0.5$. During the optimization ten variables (resistances) were chosen which changed in the range from 1 to $200 \Omega$. The number of chromosomes was three, five, seven, and 10, and the number of populations was six, eight, 11, and 26 . It was minimixed the maximum sum of the peak voltage values at preset points of the ANS PCB bus (outputs of the conductor 1, 2, and 3). Therefore, the aim of the optimization was to determine such resistance parameters, at which the sum of the highest voltages at points I, II, and III will be minimal.

The GA optimization results $\left(U_{\min }\right.$, the minimum value of the sum of peak voltages at points I, II, and III) with different runs and the number of the fitness function calculations are presented in Table 6 , where $N_{R}$ is a number of a run, and $n$ is the number of fitness function calculations. The GA was launched ten times for each combination of the chromosome number and the population number. It was made in order to check the convergence of the fitness function results. The convergence diagram of the $U_{\min }$ values with a different $n$ is shown in Figure 29. The dependences of the minimum voltage values on the $n$ are shown in Figure 30.

Table 6. $U_{\min }$ and $\mathrm{mV}$ for different $N_{r}$ and $n$.

\begin{tabular}{|c|c|c|c|c|c|c|c|c|}
\hline \multirow{2}{*}{$\mathbf{N}_{\mathbf{R}}$} & \multicolumn{8}{|c|}{$\mathbf{k}_{\mathrm{c}}$} \\
\hline & 18 & 30 & 33 & 55 & 56 & 60 & 110 & 260 \\
\hline 1 & 428 & 61 & 53 & 50 & 77 & 45 & 44 & 41 \\
\hline 2 & 82 & 87 & 283 & 52 & 154 & 47 & 765 & 35 \\
\hline 3 & 584 & 170 & 78 & 47 & 94 & 46 & 84 & 23 \\
\hline 4 & 120 & 234 & 59 & 52 & 72 & 187 & 59 & 22 \\
\hline 5 & 356 & 180 & 46 & 44 & 86 & 82 & 62 & 35 \\
\hline 6 & 105 & 236 & 47 & 59 & 143 & 52 & 44 & 26 \\
\hline 7 & 130 & 77 & 44 & 31 & 60 & 88 & 47 & 28 \\
\hline 8 & 115 & 132 & 52 & 54 & 71 & 51 & 59 & 18 \\
\hline 9 & 413 & 49 & 187 & 88 & 54 & 76 & 60 & 16 \\
\hline 10 & 122 & 144 & 154 & 37 & 38 & 72 & 54 & 35 \\
\hline
\end{tabular}




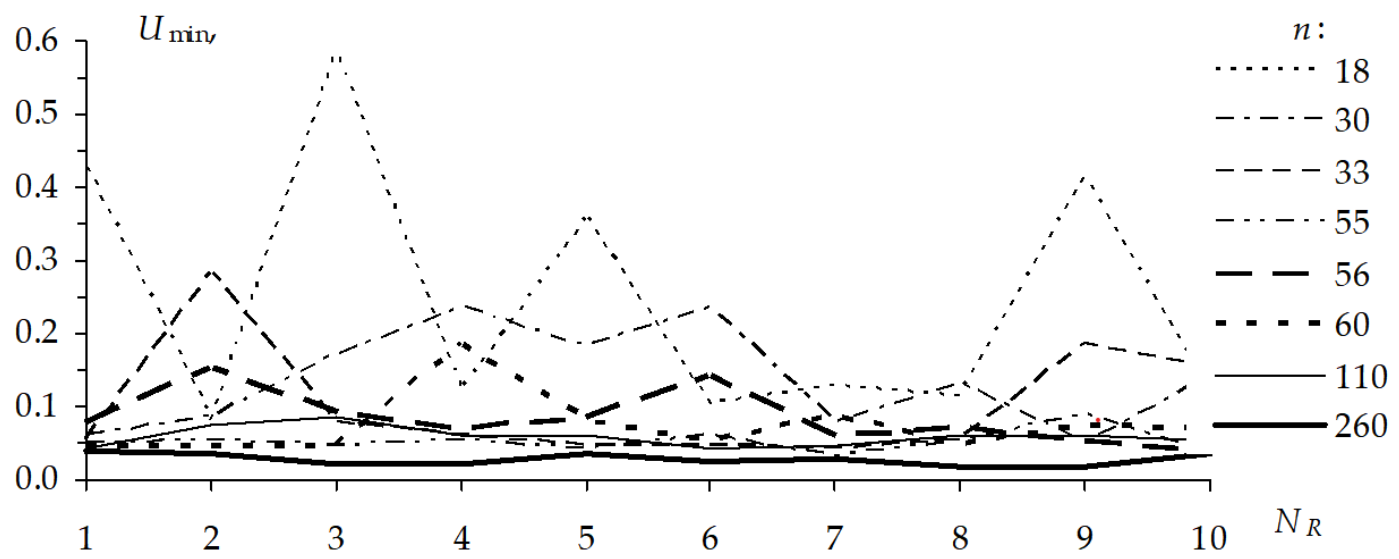

Figure 29. $U_{\min }$ values for ten runs with different $n$.

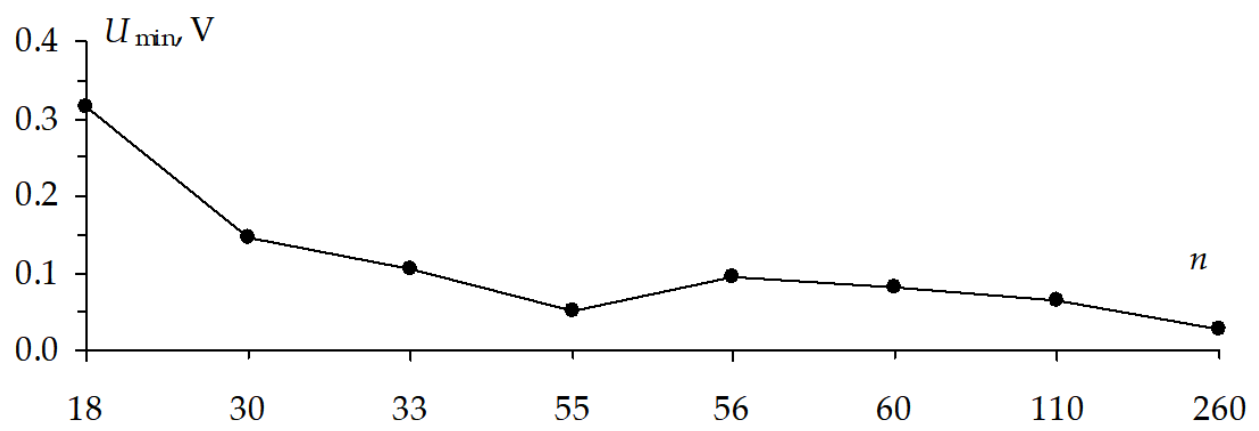

Figure 30. Dependences of the $U_{\min }$ on $n$.

The resistance values, obtained for the best fitness function result (run 9 from Table 6 when $n$ was 260), were used for calculating voltage waveforms. The waveform was calculated at the preset points with the use of these parameters (Figure 31). In addition, Figure 32 shows the voltage waveforms which are calculated in these points before the optimization.

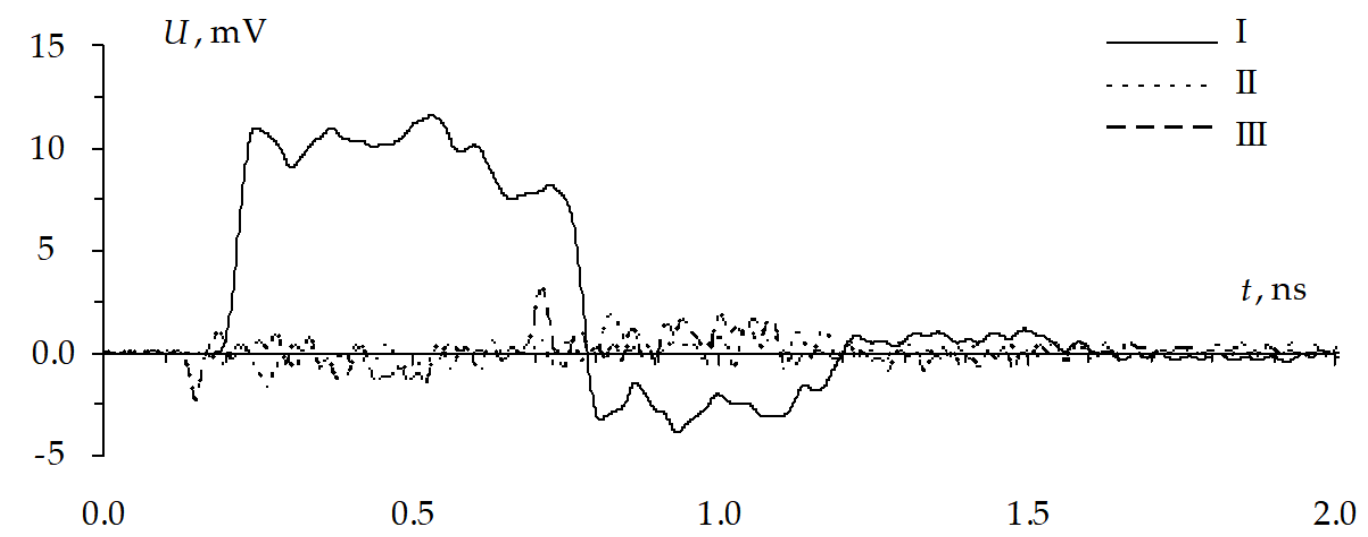

Figure 31. Signal waveforms for the best fitness function result at I, II, and III points. 


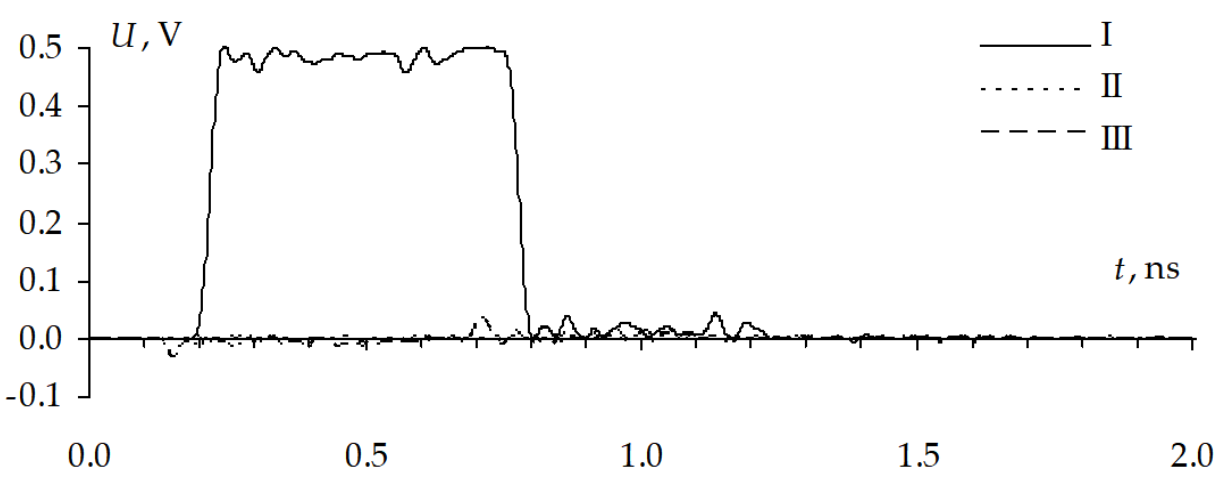

Figure 32. Signal waveforms at I, II, and III points before using the optimization.

Consider the results of the optimization from Table 6 . The result of the fitness function becomes smaller with an increase in the number of calculations (it is also shown in Figure 30). It is observed that a good convergence in the fitness function results when the number of calculations is more than 110 (Figure 29). With the maximal number of calculations, the summarized peak voltage value is $16 \mathrm{mV}$ (run 9 from Table 6) which is 30 times smaller than the signal amplitude $(0.5 \mathrm{~V})$ in the active conductor before using the optimization (Figure 32).

The significance of the optimization by means of GA to detect and localize the sum of several extreme points of a signal with a changing of load parameters is shown in this study. For example, the use of optimization helps to decrease the sum of extreme points by 30 times.

\section{Use of Evolution Strategies}

However, despite the popularity of GAs in various scientific spheres, other optimization methods are also used [42]. Therefore, it is useful to consider how the task of detecting and localizing the signal extreme points in an ANS PCB bus can be solved by an another method of optimization, for example, an evolution strategy (ES). The ESs have a parameter of initial solution (IS) in contrast to GAs, and it is useful to study the effect of the IS of ESs on the signal extreme point in a preset point of the ANS PCB bus when detecting the worst-case effects.

The goal of this study is to investigate the use of an ES and the IS influence when detecting the worst-case effects of the ultrashort pulse propagation in the PCB bus of a spacecraft ANS. The PCB fragment with the preset point is shown in Figure 33.

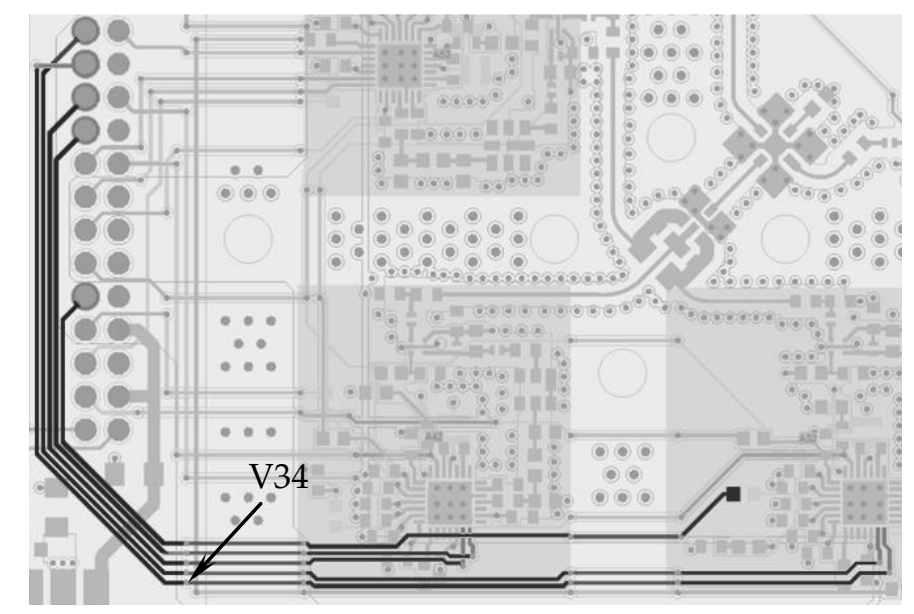

Figure 33. Bus under investigation on the PCB fragment.

The ES algorithm in general view can be formulated as [43]:

(1) The initialization of a population $\mathbf{P}_{\mu}=\left\{\mathbf{a}_{1}, \ldots, \mathbf{a}_{\mu}\right\}$ with the use of $\mu$ parent chromosomes. 
(2) The generation of $\lambda$ offspring $\mathbf{a}$ forming the offspring population $\hat{\mathbf{P}}_{\lambda}=\left\{\hat{\mathbf{a}}_{1}, \ldots, \hat{\mathbf{a}}_{\lambda}\right\}$ where each offspring $\hat{a}$ is generated by the following steps:

(3) Select (randomly) $\varrho$ parents from $P_{\mu}$ (if $\varrho=\mu$ take all parental individuals instead).

(4) Recombine the $\varrho$ selected parents to form a recombinant individual $\mathrm{r}$.

(5) Mutate the strategy parameter set $s$ of the recombinant $\mathrm{r}$.

(6) Mutate the objective parameter set, $y$, of the recombinant, $r$, using the mutated strategy parameter set to control the statistical properties of the object parameter mutation.

(7) The selection of a new parent population (using deterministic truncation selection) from either the offspring population $\hat{\mathbf{P}}_{\lambda}$ (this is referred to as comma-selection, usually denoted as " $(\mu, \lambda)$ selection"), or the offspring $\hat{\mathbf{P}}_{\lambda}$ and parent $P_{\mu}$ population (this is referred to as plus-selection, usually denoted as " $(\mu+\lambda)$ selection").

(8) Go to 2 until the termination criterion is fulfilled.

The barecmaes2.py module [43] was used with TALGAT software to perform optimization. The optimization was applied to the whole ultrashort pulse in order to get the highest extreme value of the voltage in the V34 node (shown in Figure 33). The sigma of the ES algorithm was 10 ps. The ES was launched ten times for each IS ( $3 \mathrm{~ns}, 300 \mathrm{ps}$, and $30 \mathrm{ps}$ ). The goal of the optimization was to get such duration values of the ultrashoprt pulse at which the extreme value of the voltage in the V34 node would be the highest.

The results of the ES operation are the following: the peak voltages $\left(U_{\max }\right)$ in the preset point (for ten ES runs) and the best solutions with different ISs of the ES are shown in Table 7. (Cells with the highest $U_{\max }$ for each IS are colored in yellow.) The signal waveforms calculated with IS $=3,0.3,0.03$ ns for the highest $U_{\max }$ are shown in Figure 34 .

Table 7. $U_{\max }$ Values for 10 runs of evolution strategies (ES) with different initial solutions (ISs).

\begin{tabular}{cccc}
\hline \multirow{2}{*}{ ES Run } & \multicolumn{3}{c}{ IS, $\mathbf{n s}$} \\
\cline { 2 - 4 } & $\mathbf{3}$ & $\mathbf{0 . 3}$ & $\mathbf{0 . 0 3}$ \\
\hline 1 & 428 & 61 & 53 \\
2 & 82 & 87 & 283 \\
3 & 584 & 170 & 78 \\
4 & 120 & 234 & 59 \\
5 & 356 & 180 & 46 \\
6 & 105 & 236 & 47 \\
7 & 130 & 77 & 44 \\
8 & 115 & 132 & 52 \\
9 & 413 & 49 & 187 \\
10 & 122 & 144 & 154 \\
\hline The best solution & $325.53 \mathrm{ps}$ & $330.06 \mathrm{ps}$ & $325.59 \mathrm{ps}$ \\
\hline
\end{tabular}

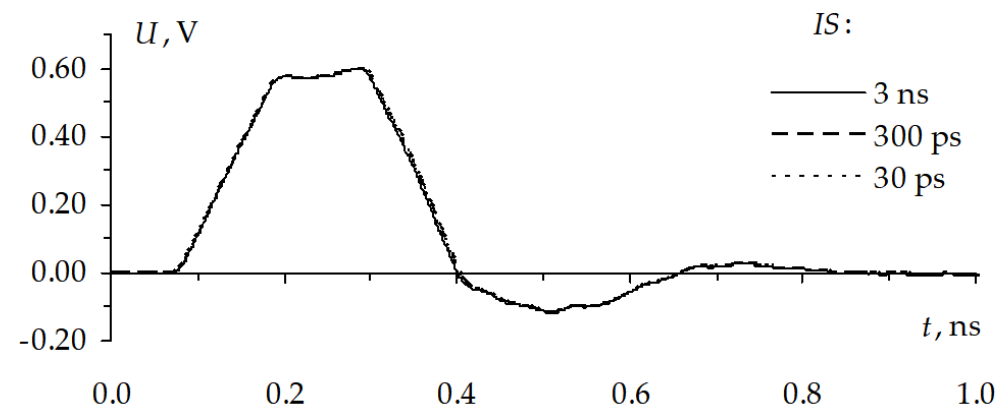

Figure 34. Voltage waveforms for the $U_{\max }$ with different ISs. 
The $U_{\max }$ arithmetic averages (of 10 runs) for IS $=3,0.3,0.03 \mathrm{~ns}$ in their dependence on the ES iteration number $\left(N_{I}\right)$ are shown in Figures 35-37, respectively. It is necessary to check the appearing of signal peaks along the whole conductor with the use of excitation parameters which have been obtained as a result of the optimization. The voltage waveforms along the active conductor are shown in Figure 38a, where the waveforms are the following: $U_{\mathrm{b}}$, at the input; $U_{\mathrm{e}}$, at the end; and $U_{\max }$, with the highest peak voltage. The voltage maximum localization is shown in Figure $38 \mathrm{~b}$. The voltage waveforms calculated along the passive (nearest to active) conductor with the highest amplitude of the crosstalk and its maximum localization are presented in Figure 39.

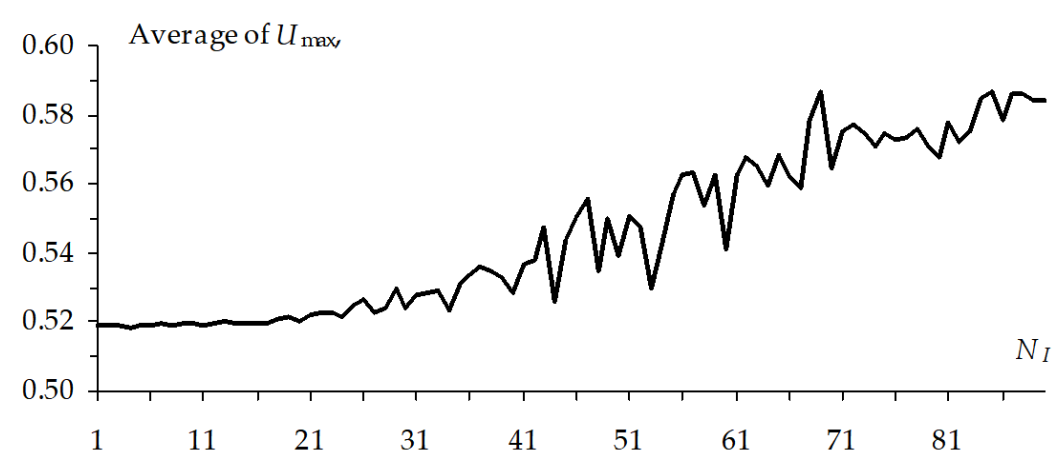

Figure 35. Dependence of the $U_{\max }$ arithmetic average on $N_{I}$ for IS $=3 \mathrm{~ns}$.

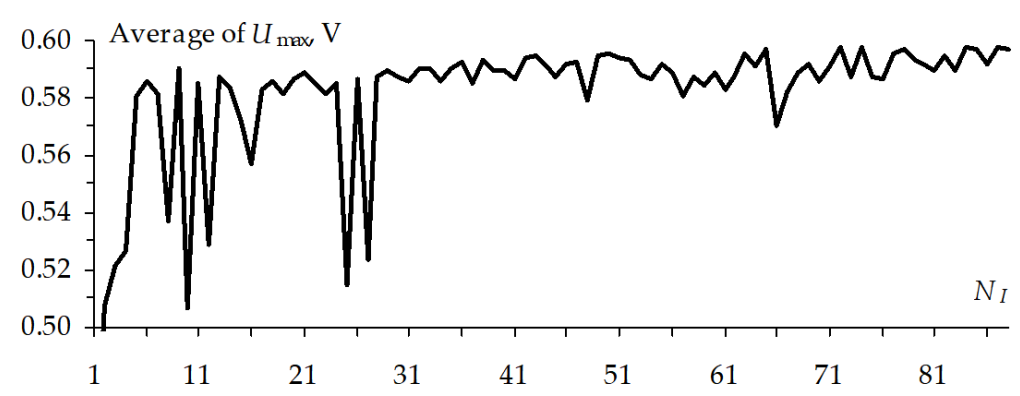

Figure 36. Dependence of the $U_{\max }$ arithmetic average on $N_{I}$ for IS $=300$ ps.

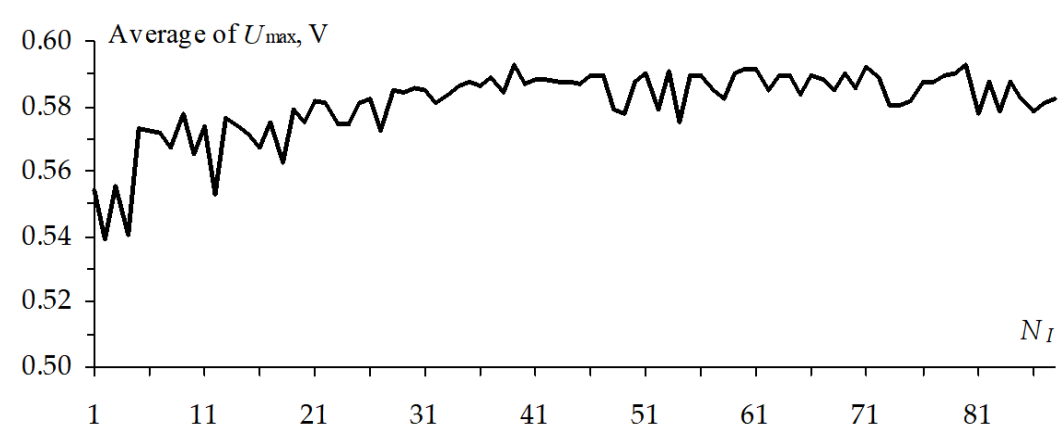

Figure 37. Dependence of the $U_{\max }$ arithmetic average on $N_{I}$ for IS $=30 \mathrm{ps}$. 


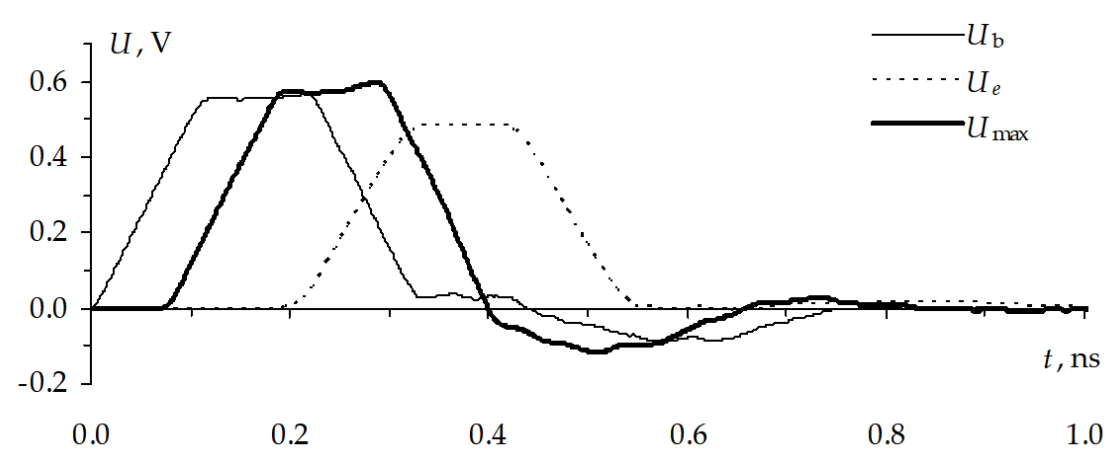

(a)

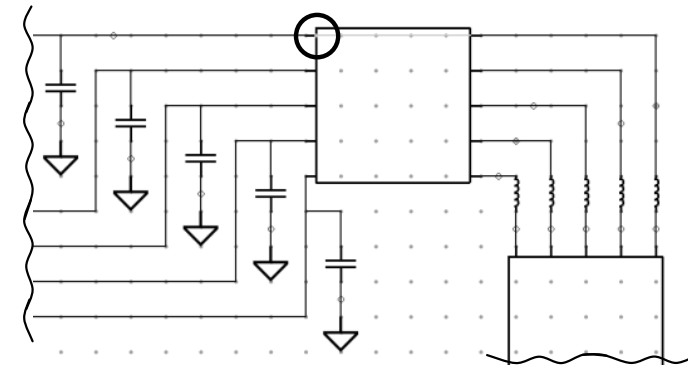

(b)

Figure 38. (a) Voltage waveforms along the active conductor; (b) the voltage maximum location.

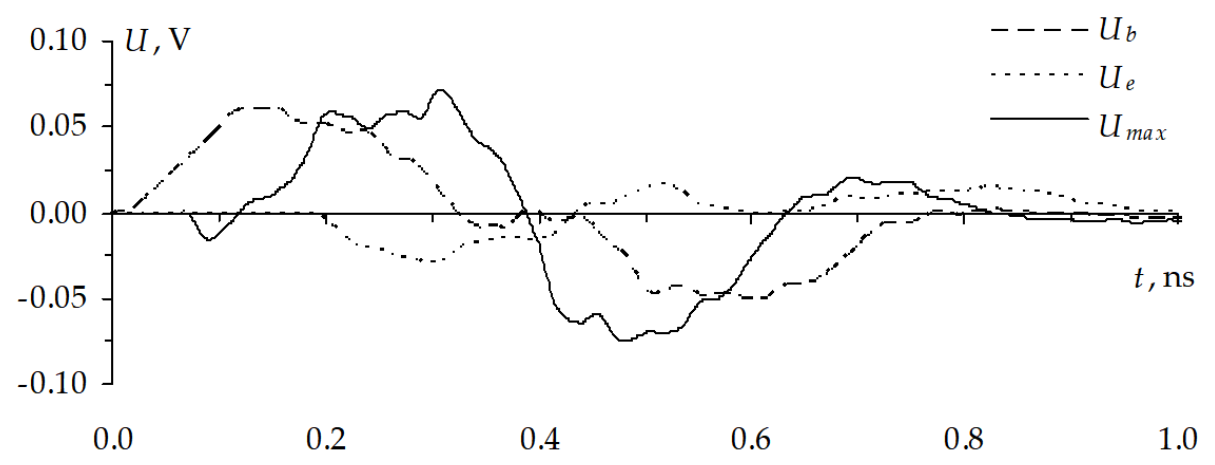

(a)

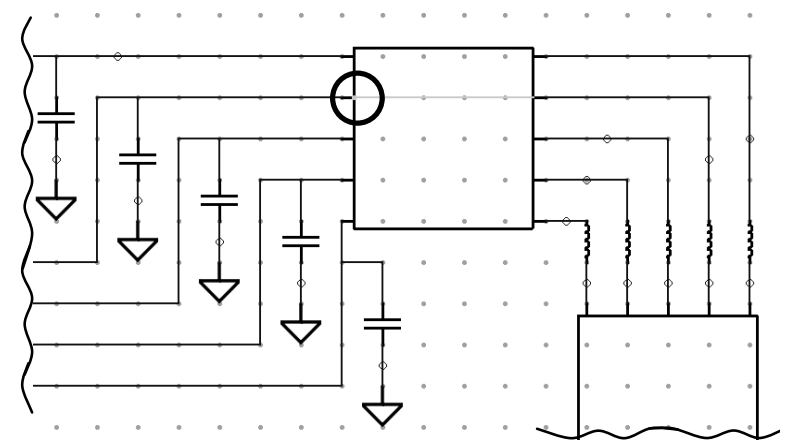

(b)

Figure 39. Voltage waveforms along the passive conductor (a) and the localization of its maximum (b).

Consider the results of the optimization presented in Table 7 . They show that all $U_{\max }$ values are very similar and differ in the fourth decimal place. The same situation is observed with the best solutions obtained after each ES cycle - the differences are near 5 ps. The highest $U_{\max }$ is obtained in the first ES run for all ISs. The small differences in the obtained results hardly change the voltage waveforms calculated in the V34 node. As we can see from Figure 34, the signal waveforms coincide. 
Consider the $U_{\max }$ arithmetic average for each IS. The strongest change of $U_{\max }$ is observed when IS $=300 \mathrm{ps}$, starting from $0.5 \mathrm{~V}$ (Figure 36). However, after 30th calculation, it becomes almost the highest and other changes are within the bounds of $30 \mathrm{mV}$. Before the 30th calculation, the $U_{\max }$ change has strong spikes, which are possibly caused by a strong mutation of an offspring. When IS $=3 \mathrm{~ns}$ (Figure 35), the $U_{\max }$ arithmetic average has a smooth rising character without strong spikes (in the range up to $30 \mathrm{mV}$ ) and starting at $0.52 \mathrm{~V}$ it reaches the maximum value in the 69th calculation, and when IS $=30 \mathrm{ps}$ (Figure 37), the $U_{\max }$ changes the least of all and starting at $0.55 \mathrm{mV}$ it reaches the maximum in the 39th calculation.

Consider the voltage waveforms with the localized maximums. The maximum is localized in segment 1 of the same MCTL section both in the active (Figure 38) and passive (Figure 39) conductors. The ultrashort pulse maximum in the active conductor is $598 \mathrm{mV}$, which is $20 \%$ higher than at the steady state level. The maximum in the passive conductor is $70 \mathrm{mV}$, which is $14 \%$ of the steady state level in the active conductor.

This investigation shows the importance of using ES for optimization for detecting and localizing signal extreme points under the excitation of the ultrashort pulse with various durations. The highest peak level of the active conductor ( $20 \%$ higher than at the steady state level) and the crosstalk of $14 \%$ of the steady state level are detected for $325 \mathrm{ps}$ ultrashort pulse. It is also shown that if the IS changes towards the best solution, it does not influence the optimization result.

\section{Conclusions}

Thus, we have developed an approach which is implemented as a special program module in TALGAT software for simulating electromagnetic compatibility problems. Various investigations into detecting and localizing signal extreme points using the approach were made as follows: in a meander line with two turns (the voltage maximum exceeding the signal amplitude at the input by 1.14 times was detected); in a microstrip cross section (the voltage maximum twice as high as the signal amplitude at the input was detected); in a PCB bus of a spacecraft ANS (the voltage maximum exceeding the steady state level by $18 \%$ was detected); using GA for optimization (the voltage maximums exceeding the steady state level by $36 \%$ and $38 \%$ were detected); using ES for optimization (the voltage maximum exceeding the steady state level by $20 \%$ was detected); and also under the ESD excitation. Using the proposed approach, the simulation was carried out over 1000 times faster, while maintaining satisfying accuracy. The obtained results show that the use of GAs and ESs allows solving various complex problems. Furthermore, such an approach minimizes the consequences of intentional and unintentional EMI on REE and increases the quality of REE diagnostics. We assume that they will use the approach for analyzing the power supply bus of a spacecraft and other structures in future. Moreover, as it is known that REE diagnostics can be made by amplitude criteria based on the $N$-norms that C. Baum proposed in 1979 [44], it seems important to combine the proposed approach with these norms in order to improve REE diagnostics.

Author Contributions: Conceptualization, T.R.G; investigation, R.R.G. and T.T.G.; writing-original draft preparation, R.R.G.; writing—review and editing, T.T.G. and T.G.G.; formal analysis, T.T.G.; supervision, T.G.G.

Funding: This research was funded by the Ministry of Science and Higher Education of the Russian Federation grant number 8.9562.2017/8.9 and the Russian Federation President grant MD-2652.2019.9.

Acknowledgments: Authors thank reviewers for valuable comments that have considerably improved the paper.

Conflicts of Interest: The authors declare no conflict of interest. 


\section{Nomenclature}

$x \quad$ A coordinate along an MCTL section

$\mathrm{V}(x) \quad$ The vector describing the voltage in $x$

$\mathrm{I}(x) \quad$ The vector describing the current in $x$

$\mathrm{S}_{V} \quad$ The matrix of modal voltages

$\mathrm{S}_{I} \quad$ The matrix of modal voltages

E0, ED The propagation matrices

$\gamma_{N_{k}} \quad$ The propagation constant

$N_{k} \quad$ A number of conductors in the $k$-th MCTL section

$l \quad$ A length of an MCTL section

C1, C2 The constant vectors describing the mode amounts

$n_{\mathrm{TLS}} \quad$ A parameter describing a number of $x$

$n \quad$ A number of MCTL sections

$\mathrm{V} \quad$ The vector describing the voltages calculated in each segment of each MCTL section along the

whole conductor

W The matrices describing the lumped memory elements of the network

$\mathrm{H} \quad$ The matrices describing the lumped memoryless elements of the network

$\mathrm{D}_{k} \quad$ The selector matrix that maps the terminal currents of the $k$-th MCTL section

$\mathrm{Y}_{k} \quad$ The conductance matrix of the $k$-th MCTL section

E The constant vector with the entries determined by the independent voltage and current sources

L The matrix of electromagnetic induction

C The matrix of electrostatic induction

$\mathrm{R} \quad$ The matrix of conductivities

G The matrices of resistances

$t r l_{\mathrm{k}} \quad$ A $k$-th MCTL section

$U_{\max } \quad$ A maximum voltage value (in the block diagrams)A waveform with the maximum voltage value (in the graphics)

A minimum voltage value (in the block diagrams)A waveform with the minimum voltage

$U_{\text {min }} \quad$ value (in the graphics)

$U_{\mathrm{b}} \quad$ A voltage waveform at the beginning of a conductor

$U_{\mathrm{e}} \quad$ A voltage waveform at the end of a conductor

\section{References}

1. Paul, C. Analysis of Multiconductor Transmission Lines; Wiley: New York, NY, USA, 2007; p. 821.

2. Cao, Y.S.; Wang, Y.; Jiang, L.; Ruehli, A.E.; Fan, J.; Drewniak, J.L. Quantifying EMI: A methodology for determining and quantifying radiation for practical design guidelines. IEEE Trans. Trans. Electromagn. Compat. 2017, 59, 1424-1431. [CrossRef]

3. Paoletti, U. On the noise path determination with the noise source modulation technique. IEEE Trans. Electromagn. Compat. 2017, 59, 1400-1407. [CrossRef]

4. Li, P.; Huang, D.; Ruan, J.; Niu, X. EM Measurements between MV switching sources and colocated sensitive circuit. IEEE Trans. Electromagn. Compat. 2015, 57, 513-521. [CrossRef]

5. Mora, N.; Vega, F.; Lugrin, G.; Rachidi, F.; Rubinstein, M. Study and classification of potential IEMI sources. Syst. Des. Assess. Notes 2014, 41, 52.

6. Gizatullin, Z.M.; Gizatullin, R.M. Investigation of the Immunity of Computer Equipment to the Power-Line Electromagnetic Interference. J. Commun. Technol. Electron. 2016, 5, 546-550. [CrossRef]

7. Gaynutdinov, R.R.; Chermoshentsev, S.F. Study immunity to disturbance of electronic system aircraft by influences of intentional ultrashort electromagnetic pulses. In Proceedings of the 2016 International Conference on Actual Problems of Electron Devices Engineering (APEDE), Saratov, Russia, 22-23 September 2016; pp. 1-6. [CrossRef]

8. Moser, K.; Andersen, A.; Dennison, J.R. Dependence of electrostatic field strength on voltage ramp rate for spacecraft materials. IEEE Trans. Plasma Sci. 2017, 45, 2036-2039. [CrossRef] 
9. Huang, L.; Gao, C.; Guo, F.; Sun, C. Lightning Indirect Effects on Helicopter: Numerical Simulation and Experiment Validation. IEEE Trans. Electromagn. Compat. 2017, 59, 1171-1179. [CrossRef]

10. Cabello, M.R.; Fernandez, S.; Pous, M.; Pascual-Gil, E.; Angulo, L.D.; Lopez, P.; Riu, P.J.; Gutierrez, G.G.; Mateos, D.; Poyatos, D.; et al. SIVA UAV: A case study for the EMC analysis of composite air vehicles. IEEE Trans. Electromagn. Compat. 2017, 59, 1103-1113. [CrossRef]

11. Wang, D.; Yang, S.; Wang, L.; Liu, W. Hardware-in-the-loop simulation for aircraft electric power system. In Proceedings of the 2016 International Conference on Electrical Systems for Aircraft, Railway, Ship Propulsion and Road Vehicles \& International Transportation Electrification Conference (ESARS-ITEC), Toulouse, France, 2-4 November 2016; pp. 1-5. [CrossRef]

12. Yuqing, L.; Tianshe, Y.; Jian, L.; Na, F.; Guan, W. A fault diagnosis method by multi sensor fusion for spacecraft control system sensors. In Proceedings of the 2016 IEEE International Conference on Mechatronics and Automation, Harbin, China, 7-10 August 2016; pp. 748-753. [CrossRef]

13. Shiue, G.H.; Shiu, J.H.; Chiu, P.W. Analysis and design of crosstalk noise reduction for coupled striplines inserted guard trace with an open-stub on time-domain in high-speed digital circuits. IEEE Trans. Compon. Packag. Manuf. Technol. 2011, 1, 1573-1582. [CrossRef]

14. Farhan, M.A.; Gad, E.; Nakhla, M.S.; Achar, R. New method for fast transient simulation of large linear circuits using high-order stable methods. IEEE Trans. Compon. Packag. Manuf. Technol. 2013, 3, 661-669. [CrossRef]

15. Hao, H.; Xia, Y. Vibration-based damage detection of structures by genetic algorithm. J. Comput. Civ. Eng. 2002, 16, 222-229. [CrossRef]

16. Cheng, X.; Ciuonzo, D.; Rossi, P.S. Multi-bit decentralized detection through fusing smart \& dumb sensors based on rao test. IEEE Trans. Aerosp. Electron. Syst. 2019. [CrossRef]

17. Moreno, L.; Armingol, J.M.; Garrido, S.; de la Escalera, A.; Salichs, M.A. A genetic algorithm for mobile robot localization using ultrasonic sensors. J. Intell. Robot. Syst. 2002, 34, 135-154. [CrossRef]

18. Sardellitti, S.; Scutari, G.; Barbarossa, S. Joint optimization of radio and computational resources for multicell mobile-edge computing. IEEE Trans. Signal Inf. Process. Netw. 2015, 1, 89-103. [CrossRef]

19. Pazos, S.; Hurtado, M.; Muravchik, C.H.; Nehorai, A. Projection matrix optimization for sparse signals in structured noise. IEEE Trans. Signal Process. 2015, 63, 3902-3913. [CrossRef]

20. Rubio, J.; Iserte, A.P.; Palomar, D.P.; Goldsmith, A. Joint optimization of power and data transfer in multiuser MIMO systems. IEEE Trans. Signal Process. 2016, 65, 212-227. [CrossRef]

21. Yin, F.; Zhao, Y.; Gunnarsson, F.; Gustafsson, F. Received-signal-strength threshold optimization using gaussian processes. IEEE Trans. Signal Process. 2017, 65, 2164-2177. [CrossRef]

22. Denisova, L.A.; Meshcheryakov, V.A. Control system synthesis based on multicriteria optimization using genetic algorithm. In Proceedings of the 2017 Dynamics of Systems, Mechanisms and Machines (Dynamics), Omsk, Russia, 14-16 November 2016; pp. 1-5.

23. Mohammed, H.J.; Abdulsalam, F.; Abdulla, A.S.; Ali, R.S.; Alhameed, R.A.A.; Noras, J.M.; Abdulraheem, Y.L.; Ali, A.; Rodriguez, J.; Abdalla, A.M. Evaluation of genetic algorithms, particle swarm optimisation, and firefly algorithms in antenna design. In Proceedings of the Synthesis, Modeling, Analysis and Simulation Methods and Applications to Circuit Design (SMACD), Lisbon, Portugal, 27-30 June 2016; pp. 1-4.

24. Goudos, K.; Kalialakis, C.; Mittra, R. Evolutionary algorithms applied to antennas and propagation: A review of state of the art. Int. J. Antennas Propag. 2016, 2016, 1010459. [CrossRef]

25. Cheng, Y.S.; Wu, R.B. Direct eye diagram optimization for arbitrary transmission lines using fir filter. IEEE Trans. Compon. Packag. Manuf. Technol. 2011, 1, 1250-1258. [CrossRef]

26. Kwak, N.S.; Lee, J.; Jang, Y.H. Genetic-algorithm-based controlling of microcontact distributions to minimize electrical contact resistance. IEEE Trans. Compon. Packag. Manuf. Technol. 2012, 2, 1768-1776. [CrossRef]

27. Zhang, M.S.; Tan, H.Z.; Mao, J.F. Signal-integrity optimization for complicated multiple-input multipleoutput networks based on data mining of s-parameters. IEEE Trans. Compon. Packag. Manuf. Technol. 2014, 4, 1184-1192. [CrossRef]

28. Liu, H.J.; Jain, R.; Rajawat, K. Asynchronous decentralized dynamic optimization for power network voltage control. IEEE Trans. Signal Inf. Process. Netw. 2016, 3, 568-579. [CrossRef]

29. Chung, C.; Lee, S.; Kwak, B.M.; Kim, G.; Kim, J. A delay line circuit design for crosstalk minimization using genetic algorithm. IEEE Trans. Comput. Aided Des. Integr. Circuits Syst. 2008, 27, 578-583. [CrossRef] 
30. Gazizov, R.R.; Zabolotsky, A.M.; Orlov, P.E. Signal maximum localization in multiconductor transmission lines of printed circuit boards using TALGAT system. Dokl. Tom. Gos. un-ta System upr. i Radioelectroniki 2015, 38, 147-150. (In Russian)

31. Gazizov, R.R.; Zabolotsky, A.M.; Gazizov, T.T. Research on ultrashort pulse propagation in microstrip C-section with variated separation between coupled conductors. Dokl. Tom. Gos. un-ta System upr. i Radioelectroniki 2016, 19, 79-82. (In Russian)

32. Gazizov, R.R.; Zabolotsky, A.M.; Gazizov, T.T.; Belousov, A.O. Influence of ultrashort pulse duration on its peak values localization in PCB of spacecraft autonomous navigation system. In Proceedings of the 18th International Conference of Young Specialists on Micro/Nanotechnologies and Electron Devices, Erlagol, Russia, 29 June-3 July 2017; pp. 69-74. [CrossRef]

33. Gazizov, R.R.; Belousov, A.O.; Gazizov, T.T. Influence of ultrashort pulse duration on localization of crosstalk peak values in PCB of spacecraft autonomous navigation system. In Proceedings of the International Siberian Conference on Control and Communications (SIBCON 2017), Astana, Kazakhstan, 29-30 June 2017; pp. 1-6. [CrossRef]

34. Gazizov, R.R.; Belousov, A.O.; Gazizov, T.T.; Gazizov, T.R. Optimization of ultrashort pulse duration with usage of genetic algorithms by criteria of peak voltage maximization in PCB bus. In Proceedings of the IEEE 2017 Siberian Symposium on Data Science and Engineering (SSDSE), Novosibirsk, Russia, 12-13 April 2017; pp. 1-5. [CrossRef]

35. Gazizov, R.R.; Ryabov, R.S.; Gazizov, T.T. Influence of crossover and mutation coefficients on GA optimization of ultrashort pulse duration by criteria of peak voltage maximization in PCB bus. In Proceedings of the IEEE 2017 International Multi-Conference on Engineering, Computer and Information Sciences (SIBIRCON), Novosibirsk, Russia, 18-24 September 2017; pp. 415-420. [CrossRef]

36. Zabolotsky, A.; Gazizov, T. Time Response of Multiconductor Transmission Lines; Tomsk State University: Tomsk, Russia, 2007; p. 152.

37. Kuksenko, S.P.; Gazizov, T.R.; Zabolotsky, A.M.; Ahunov, R.R.; Surovtsev, R.S.; Salov, V.K.; Lezhnin, E.V. New developments for improved simulation of interconnects based on method of moments. Advances in Intelligent Systems Research. In Proceedings of the 2015 International Conference on Modeling, Simulation and Applied Mathematics, Phuket, Thailand, 23-24 August 2015; pp. 293-301. [CrossRef]

38. Gazizov, R.R. Simulation of ESD effects on PCB bus of spacecraft autonomous navigation system. In Proceedings of the IEEE 2017 International Multi-Conference on Engineering, Computer and Information Sciences (SIBIRCON), Novosibirsk, Russia, 18-24 September 2017; pp. 501-505. [CrossRef]

39. Gazizov, T.R.; Dolganov, E.S.; Zabolotsky, A.M. Modal filter as a device for electrostatic discharge protection of onboard computers and control units of space vehicles. Russ. Phys. J. 2012, 55, 282-287. [CrossRef]

40. IEC 61000-4-2 (2003). Electromagnetic Compatibility (EMC) Part 4: Testing and Measurement Techniques-Section 2: Electrostatic Discharge Immunity Test; IEC: Geneva, Switzerland, 2003.

41. Gazizov, R.R.; Kuharenko, M.N.; Gazizov, T.R. Optimization of PCB bus loads with usage of genetic algorithms by criteria of peak voltage minimization. In Proceedings of the 2017 Dynamics of Systems, Mechanisms and Machines (Dynamics), Omsk, Russia, 14-16 November 2017; pp. 1-5. [CrossRef]

42. Beyer, H. Evolution strategies. Scholarpedia 2007, 2, 1965. [CrossRef]

43. Hansen, N. Python: Module Barecmaes2. Lri.fr. 2017. Available online: https://www.lri.fr/ \{\}hansen/ barecmaes2.html (accessed on 17 November 2017).

44. Baum, C. Norms and Eigenvector Norms. Math. Notes 1979, 63, 1-42.

(C) 2019 by the authors. Licensee MDPI, Basel, Switzerland. This article is an open access article distributed under the terms and conditions of the Creative Commons Attribution (CC BY) license (http://creativecommons.org/licenses/by/4.0/). 